\title{
SUCCESSION OF XERIC CALCAREOUS GRASSLAND TOWARD THERMOPHILOUS OAK FOREST: THE CASE OF ABAVA VALLEY, WEST LATVIA
}

\author{
Māris Laiviṇš ${ }^{1}$, Agnese Priede ${ }^{2, \#}$, Dārta Kaupe ${ }^{1}$, and Andis Lazdinšs ${ }^{1}$ \\ ${ }^{1}$ Latvian State Forest Research Institute "Silava", 111 Rīgas Street, Salaspils, LV-2169, LATVIA, maris.laivins@silava.Iv \\ ${ }^{2}$ Nature Conservation Agency, Baznīcas Street 7, Sigulda, LV-2150, LATVIA \\ \# Corresponding author, agnesepriede@ hotmail.com
}

Communicated by Tālis Gaitnieks

\begin{abstract}
The succession of semi-natural xeric calcareous grassland plant communities toward deciduous forest communities is poorly studied in Latvia. There is insufficient knowledge on the natural transformation of dry calcareous grasslands of Festuco-Brometea into thermophilous quasi-climax oak forest communities of Quercetea pubescentis that are very rare in Latvia. In this paper, a geobotanical study is presented that included studies of soils, tree age, spatial pattern of trees, vegetation composition of different succession stages, and analysis of environmental factors. The study was conducted in the Abava Valley, West Latvia, an area that is unique with species-rich xeric calcareous Festuco-Brometea grasslands, thermophilous oak forests dominated by Quercus robur, and intermediate secondary succession stages with young to medium-aged Juniperus communis and Pinus sylvestris formations.
\end{abstract}

Keywords: Festuco-Brometea, Quercetea pubescentis, Juniperus communis stage, Pinus sylvestris stage, vegetation dynamics.

\section{INTRODUCTION}

Thermophilous oak forests belonging to the class Quercetea pubescentis Doing-Kraft ex Scamoni 1959 (syn. Quercetea pubescenti-petraeae Jakucs 1960), order Quercetalia pubescenti-petraeae Klika 1933, have a wide distribution range and are found from the warm Mediterranean in South Europe to the cool temperature nemoral zone in Central Europe and East Europe. These are oak-dominated forests, usually dominated by Quercus pubescens or $Q$. petraea, rarely by $Q$. robur. They usually occur on south-facing base-rich slopes on limestone or dolomite bedrock. The shrub layer and herbaceous vegetation in such oak forests are rich in subcontinental species and often are rich in rare plant species. They include four alliances: Quercion petraeae Issler 1931, Quercion pubescenti-petraeae Br.-B1 1932, Aceri tatarici-Quercion Zolyomi 1957, and Lathyro pisiformis-Quercion roboris Solomeshch et Grigoriev in Wilner et al. 2015 (Chytrý, 1997; Goncharenko et al., 2020).

The distribution of thermophilous oak forests was summarised by Goncharenko et al. (2020). Their distribution range covers the Balkan and Apenine peninsulas, France, northern part of Spain, Czechia, Slovakia, Poland, Germany, Austria, Hungary, Romania, Belarus, Ukraine, steppe and forest steppe zone in Russia reaching the south-eastern part of Urals. In some parts of the range, they are abundant, while elsewhere, e.g., in West and Central Europe, they are considered relict and scattered and occur on meso-climatically and topographically confined areas (Chytrý, 1997; Bulokhov and Semenishchenkov, 2008; Goncharenko et al., 2020). Loss of these forests was caused by massive deforestation in the Neolithic Age (Rybníèková, 1985 in Chytrý, 1997). Presence and extent of this forest community in Eastern Europe (Belarus, Ukraine, Russia) is insufficiently studied and the syntaxonomy of the communities is not always clear. In scientific literature, Latvia falls out of the currently known range, as there is a lack of studies and published data.

However, the presence of Quercetea pubescentis forest communities in Latvia is mentioned as early as in 2001 by Jermacāne \& Laiviņš (2001). Later, the community Lathyro nigri-Quercetum roboris (belonging to Quercetalia pubescenti-petreae order) was recorded and described by 
Laiviņš (2014; 2017), including the distribution map (Laiviňš, 2017), and incorporated into the list of rare habitat types in Latvia (Lathyro nigri-Quercetum roboris association) (National Regulation of the Cabinet of Ministers No. 350, issued in 20.06.2017). Two other communities under Quercetalia pubescenti-petreae have been distinguished in Latvia: Brachypodium pinnatum-Quercus robur community and Corylus avellana-Quercus robur community (Jermacāne and Laiviņ̌s, 2001; Laivin̦š, 2014).

Such forests considerably differ from the oak communities belonging to the class Querco-Fagetea that are more frequent in Latvia and elsewhere in Eastern Europe outside the steppe and forest steppe zone. Thermophilous oak forests of Quercetea pubescentis represent edaphic conditions and plant assemblages differing from the more frequent European temperate summer-green deciduous forests of Querco-Fagetea. Also, the succession and plant species composition of thermophilous oak communities, though sharing some similarities, are different from the communities of Querco-Fagetea.

The succession of xeric calcareous grassland plant communities toward deciduous oak forest communities is poorly studied in Latvia. Therefore, we conducted a geobotanical study to understand the complex of xeric calcareous grassland, scrub and thermophilous oak forest plant communities and their transformation process. The Abava Valley chosen as the study area is one of a few areas in Latvia where thermophilous oak forest communities of Quercetea pubescentis have been recorded (Laiviņš, 2014). Thermophilous oak forests may be considered azonal vegetation in Latvia, i.e., occur outside the main range. This type of forest vegetation is very rare in Latvia, as suitable edaphic and local climate conditions are very rare.

The aim of the study was to identify and describe the succession stages of xeric semi-natural grassland (Filipendula vulgaris, Helictotrichon pratense) toward scrub facies and forest pioneer communities (Juniperus communis, Pinus sylvestris) that finally lead to a relatively stable quasiclimax forest community dominated by Quercus robur. The paper contributes to the overall understanding of the distribution of thermophilous oak forests and the succession from grassland to quasi-climax forest community.

\section{MATERIALS AND METHODS}

Study area. The Vegi study area lies in the Abava Valley (Fig. 1), West Latvia, in Veǵi, 5705'05.2" N, $22^{\circ} 28^{\prime} 30.9^{\prime \prime}$ E. According to the Köppen-Geiger climate classification (Peel et al., 2007), the whole of Latvia is located in the warm-summer humid continental climate zone $(D f b)$. The study area occurs in the Abava Valley Nature Park, a protected nature area (Natura 2000).

The network of some largest rivers in Latvia is defined by regional tectonic faults in the pre-Quaternary bedrock with length of several dozen kilometres. Therefore, in river val-

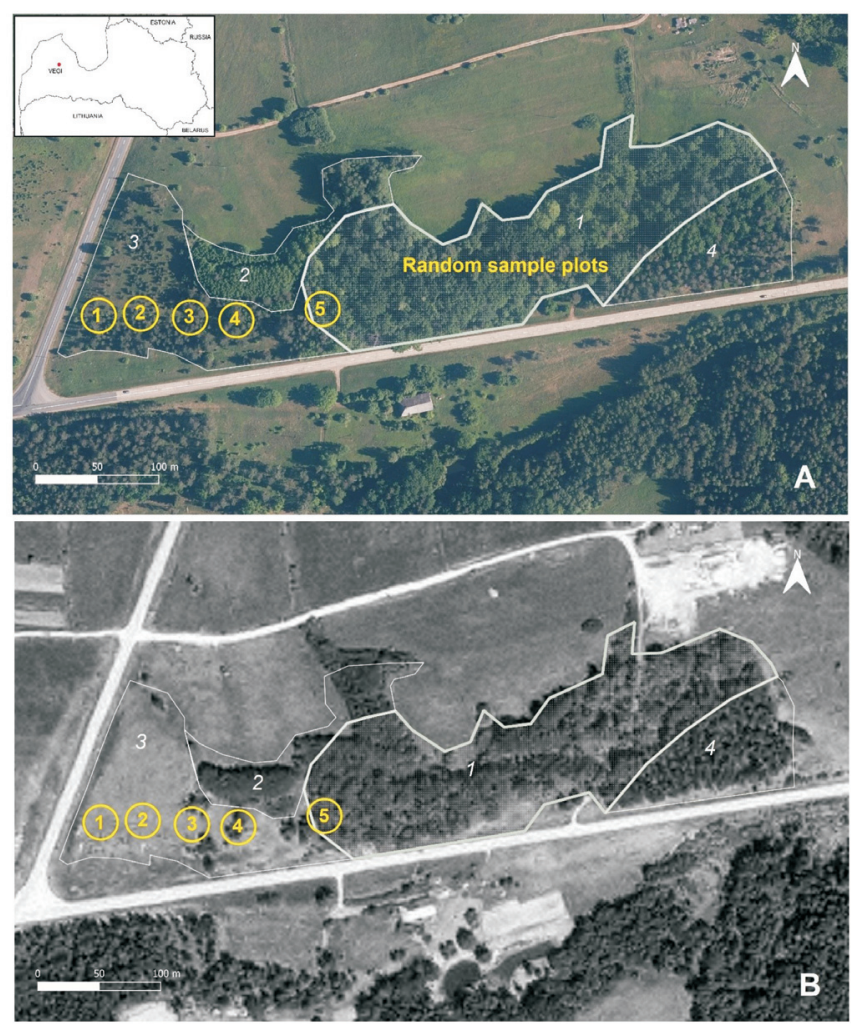

Fig. 1. Study area and location of sample plots on an orthophoto map of 2019 (A) and 1994, shortly after abandonment (B). Circled numbers (yellow) - sample plots from 2020 (Vegi1-Vegi5); forest compartments (white numbers in italic): 1 - mature oak forest (random sample plots from 2003-2020), 2 - medium-aged aspen stand, 3 - medium-aged Scots pine-juniper stand (overgrowing semi-natural grassland), 4 - medium-aged Scots pine stand. The numbers of sample plots in Table 2 (results) are as follows: Vegi $1=66$, Vegi $2=67$, Vegi $3=68$, Vegi4 $=69$, Vegi5 $=70$; the others were randomly located in forest compartment 1. Background: orthophoto map by the Latvian Geospatial Information Agency, 1994 and 2019.

leys, often Devonian carbonatic bedrock, such as dolomite and dolomitic marlstone, may be exposed or lies close to the soil surface (Eberhards, 2013; Krievāns et al., 2018). The Abava River Valley with its tributaries are among the few examples in West Latvia, where carbonatic bedrocks are exposed, which occur in a ca. 30-km long stretch between Kandava and Renda. In this stretch, the depth of the valley reaches up to $58 \mathrm{~m}$, and dolomite is exposed or lies close to the soil surface on the terraced valley slopes.

The Abava River is a 134-km long medium-sized river. At its lower and upper reaches, the river valley is less pronounced, with gentle slopes, while the middle stretch of the valley has relatively steep, terraced slopes (relative height $30-58 \mathrm{~m}$ ), oriented in an east-west direction. The valley was eroded in the pre-Quaternary period, and the slopes are formed by carbonatic rocks (dolomite, dolomitic marlstone, sandstone, carbonatic deposits with clay layers, etc.) exposed as cliffs in a few sites, but mostly covered with a shallow soil layer. On the slopes and terraces, the soils are eroded, rich in carbonates, shallow, laying on dolomite or dolomitic marlstone (Stinkulis and Sinkule, 2013; Strautnieks, 2020). This type of soil occurred also in the study area. 
The shallow, carbonatic soils on dolomite and dolomitic marlstone and local climate have played important roles in the development of the particular flora and vegetation in the Abava Valley (Tabaka and Klavina, 1981; Priede, 2009). The dry, carbonatic soils host xeric calcareous plant communities, such as semi-natural grasslands with Filipendula vulgaris, Helictotrichon pratensis, and Astragalus danicus; forest fringe plant communities with Geranium sanguineum, Laserpitium latifolium, and Origanum vulgare; scrubs with Juniperus communis, Corylus avellana, Rhamnus cathartica, Berberis vulgaris, and Prunus institia; coniferous forests with Pinus sylvestris, Brachypodium pinnatum, Festuca ovina, F. trachyphylla, and Primula veris; and deciduous broadleaved forests with Quercus robur, Lathyrus niger, Viola mirabilis, and Convallaria majalis. In some areas with discharge of carbonate-rich groundwater, alkaline fens with Schoenus ferrugineus and Carex davalliana have developed (Tabaka and Klavina, 1981; Rūsiņa, 2007).

In the Abava Valley, the xeric calcareous plant communities (semi-natural grasslands, forests, and intermediate scrub facies) cover relatively small areas. Their mosaic-type spatial pattern is influenced by land use and land use intensity in the past; they represent various stages of succession. The transition zones between various succession stages are usually gradual, and therefore many of the characteristic species occur at different succession stages, thus forming a syntaxonomic continuum. The total number of plant species that form various communities is related to the succession stage and the mutual spatial and temporal relation with other phytocoenoses.

The river valley near Vegit, the study area, is $40 \mathrm{~m}$ in depth and asymmetric, as the relative height of the south-facing slope is larger in comparison to the gentler, lower northfacing slope (Krievāns et al., 2018; Strautnieks, 2020). The study area (ca. 3 ha) is located on the upper part of the south-facing valley slope on dolomite rock close to the soil surface (50-60 cm below the soil surface, with 20-30\% admixture of dolomite and dolomitic marlstone pebbles in the topsoil). At the study area, the slope inclination varies from 7 to 9 degrees, and exposition is 180 degrees. The altitude of the study area varies between 58 and $64 \mathrm{~m}$ a. s. 1 .

The climate in the region is relatively mild and moist with an average annual temperature of $+6.3{ }^{\circ} \mathrm{C}$ (here and further the climate data from the period 1981-2021 are summarised from the nearest meteorological station in Stende, $7 \mathrm{~km}$ from the study area). The coldest month is February with $-3.3{ }^{\circ} \mathrm{C}$ on average; the average temperature in July is $+16.9{ }^{\circ} \mathrm{C}$. The annual precipitation sum is $724 \mathrm{~mm}$. The vegetation period is about 196 days (Briede, 2018). Overall, the micro-climate in the valley is more continental than in the surrounding areas. The south-facing slopes (including Veği site) are more exposed to solar radiation and receive a larger amount of light and warmth than the north-facing slopes. On the slopes, there is increased runoff, enhanced moisture deficit in the soils and increased evaporation. The air temperatures tend to be lower on the valley floor and are higher in the upper part of the slopes; the difference in tem- peratures can be even $7-8{ }^{\circ} \mathrm{C}$ (Temnikova, 1958; Strautnieks, 2020).

The valley is distinct with mosaic-type landscapes, richness of flora, especially flora related to dry calcareous grassland communities, thermophilous forest fringe and deciduous broad-leaved forest plant communities occurring on the slopes, terraces and floodplain of the valley (Tabaka and Klavina, 1981; Rūsiņa, 2007).

The study area (Fig. 1) represents a series of vegetation succession: (1) abandoned semi-natural xeric grassland partly overgrown with secondary, sparse tree cover dominated by common juniper Juniperus communis and Scots pine Pinus sylvestris; (2) young to medium-aged woodlands dominated by Pinus sylvestris and aspen Populus tremula; and (3) mature pedunculate oak Quercus robur forest.

The history of land use in the study site is not known in detail but can be partly reconstructed by "reading the landscape" and combining it with general knowledge on the land use history in Latvia and in the particular region and maps. The topographic maps of the early and mid-20th century depict the current oak forest patch in its contemporary borders. The grassland area may have been used as a hay meadow and pasture in the past (indicated by some residual fence fragments found during the fieldwork). It is highly probable that, similar to the common practice in the early $20^{\text {th }}$ century Latvia (Lazdiňš, 1939; Dumpe, 1999), 80-100 years ago, the oak forest was grazed. Later, the grazing practice may have ceased; still, the forest may have been used for fuelwood collecting (e.g., removal of deadwood and fallen branches). Management of the grassland ceased at least 30 years ago.

According to the National Forest Register (State Forest Service, Latvia), the age of the oak forest is 135 years (in fact, it is older as shown further by our study results), whereas the younger forest on the transitional zone between the overgrowing grassland and the old-growth oak stand varies from 45 (aspen stand) to 65 (pine stand) years (Figure 1).

Vegetation sampling. In the study area, there are young forest stages of various age $(<100$ years), in a mosaic with open grassland patches and a mature forest with Quercus robur. To characterise the succession stages from open grassland to oak forest, in June 2020 a transect with five sample plots was established (Vegi1, Vegi2, Vegi3, Vegi4, Vegi5) (Fig. 1). Four sample plots (Vegi1-Vegi4) were established in the young pine forest: circle-shaped plots with radius $10.0 \mathrm{~m}$, area $314.0 \mathrm{~m}^{2}$; in the oak forest - a circleshaped plot (Vegi5) with radius $15.0 \mathrm{~m}$ (area $706.5 \mathrm{~m}^{2}$ ). Different plot sizes were used to cover the diversity of spatial structures in the forest stand. The forest stand structure of younger forest stages typical for the area could be covered by a smaller plot size $\left(314.0 \mathrm{~m}^{2}\right)$ that provides more precise estimation of the cover of vegetation in all layers and individual species. A larger plot size $\left(706.5 \mathrm{~m}^{2}\right)$ was chosen to include a sufficient number of mature oaks, which 
is essential to understand the spatial structure of the forest; otherwise the smaller size plots would include only 2-3 trees. Although usually in larger plots the accuracy of vegetation cover assessment is lower than in smaller plots, this was chosen as a compromise to be able to estimate the vegetation structure in all layers. The plot sizes were also defined by the size of the study area, i.e., the transect across the young forest stage did not exceed $200 \mathrm{~m}$ and included a series of succession stages that could not be well represented by larger sample plots, i.e., it was important to avoid partial overlapping of sample plots. The centre of each plot was marked with a wooden pole $(1 \mathrm{~m}$ tall, diameter $5-10 \mathrm{~cm})$.

All plant species and their cover (\%) were recorded in each layer (E3 - trees, E2 - shrubs, E1 - herbs, E0 - bryophytes) (Dierschke, 1994). The cover was estimated visually by experienced surveyors.

To characterise the quasi-climax oak forest community, we used relevés from earlier years (2003-2010) from the same site, in total 15 (Fig. 1). Here, the vegetation was described using randomly located sample plots with sizes varying from 350 to $700 \mathrm{~m}^{2}$ (the size for each plot is given in Table 2) and using the same vegetation recording method as described above. In contrast to the five above mentioned plots from 2020 (Vegi1-Vegi4), in the earlier sampled plots only vegetation was recorded, but no measurements of tree dimensions, annual rings or spatial distribution were done.

The nomenclature of taxa follows Gavrilova and Šulcs (1999) for vascular plants, Ābolin,a et al. (2016) for mosses. The nomenclature of vegetation syntaxa follows regional vegetation reviews: Mucina et al. (1993), Rūsinga (2003, 2007), Wilner and Grabherr (2007), Chytrý and Tichý (2008), Chytrý (ed.) (2013).

Measurements of tree dimensions, spatial pattern of trees and annual rings. In each sample plot within the transect Vegi1-Vegi5, each tree taller than $3 \mathrm{~m}$ (in the young pine forest) or $5 \mathrm{~m}$ (in the mature oak forest) was marked with a unique number. For each marked tree the following parameters were recorded:

- Diameter at breast height $1.30 \mathrm{~m}$ (measured using Hultafors Talmeter $3 \mathrm{~m}$ );

- The height of trees (measured using Vertex IV, Haglöf Sweden);

- Wood cores from each living tree within the sample plots Vegi1-Vegi5 were taken using a Presler border for analysis of annual rings and annual increment; the radial growth was measured using LINTAB IV, and data were processed by TSAP Win Scientific 0.55 and MS Excel software;

- The distance of each tree and its azimuth from the plot centre were measured using a Vertex IV and a Suunto compass. For each tree, considering its location within the sample plot, the nearest neighbour index (NNI) was used. NNI is a tool to measure precisely the spatial distribution of a pattern and see if it is regular, random or clustered (intermediate patterns are possible). The distance to the nearest neighbour of each tree within the plot was measured using ArcView 9.0 software. Then, the distribution of distances was tested using the z-score. At a 95\% confidence level, a z-score within the interval -1.96 to 1.96 confirms the null hypothesis, i.e., the spatial distribution is random. If the $\mathrm{z}$-score is $>1.96$, the spatial pattern is regular, and if the $\mathrm{z}$-score is $<-1.96$, the spatial distribution is clustered (Liepa, 1974).

Soil sampling and analysis. Soil was sampled from each of the transect plots Vegi1-Vegi5, but not in the earlier recorded relevés plots from 2003-2010. In the mature oak forest, the soil samples were collected only in Vegi5, as other sample plots were very similar to each other in vegetation pattern and composition. The soil data from Vegi5 were further extrapolated to the entire oak forest stand in the study area. The soil was taken at the depth of $1-15 \mathrm{~cm}$ and 15-30 cm (three sub-samples at each depth) and a mixed sample from each plot. The fine earth fraction of soils $(\mathrm{D}<2 \mathrm{~mm}$ ) was used for chemical analysis. Soil exchangeable acidity $(\mathrm{pH})$ was determined using potassium chloride $(1 \mathrm{M})$, the contents of adsorbed bases and base saturation was determined in $0.1 \mathrm{M}$ hydrochloric acid solution using titrimetry, calcium $(\mathrm{Ca})$, magnesium $(\mathrm{Mg})$, potassium $(\mathrm{K})$, iron $(\mathrm{Fe})$ and manganese $(\mathrm{Mn})$ was determined in $0.1 \mathrm{M}$ hydrochloric acid solution using atomic emission or absorption spectroscopy.

During the fieldwork, all soil depths were examined by applying hydrochloric acid $10 \% \mathrm{HCl}$ to detect the presence of free carbonates. Free carbonates were used as an additional indicator in characterisation of the soils.

Data analysis. The vegetation data were entered into the Turboveg database (Hennekens and Schaminée, 2001) and then exported for further analysis. The relevés were analysed using two-way cluster analysis in PC-ORD 6.0, and the clusters were distinguished using the flexible beta method, where the beta value was -0.25 (McCune and Mefford, 2002; 2011). To compare the similarity of relevés using plant cover as an indicator, the Sørensen coefficient was calculated.

Detrended Correspondence Analysis (DCA) (PC-ORD 6.0; McCune and Mefford, 2002; 2011) was applied (rescaling the axes without downweighting rare species). In detrending by segments the default option (26 segments) was applied. Kendall correlation coefficients between the environmental variables and the DCA Axes 1 and Axis 2 were calculated and used for descriptive purposes.

Vegetation data (20 relevés) were used as the first matrix, the Ellenberg indicator values (light, temperature, continentality, moisture, reaction, nitrogen) for Central Europe (Ellenberg et al., 1992) were used as the second data matrix in the DCA ordination. Weighted averages of all indicator 
values were calculated with weights determined by the abundance of each species in the relevé.

To analyse the mean values for each sample plot, standard error and standard deviation were calculated, and the confidence level of the variation among the sample plots was tested using t-test (MS Excel, Data Analysis tool).

\section{RESULTS}

Physical and chemical properties of soil. The physical and chemical properties of soil in the study area were characterised by granulometric composition, soil exchangeable acidity, base saturation, organic matter, and total nitrogen and macroelement concentration in two topsoil horizons (at the depth up to $15 \mathrm{~cm}$ and at depth of $15-30 \mathrm{~cm}$ ). The variation in physical and chemical properties at all five soil sampling sites (Vegi1-Vegi5) was negligible (Table 1).

The examined soil layer contained three particle size fractions: medium sand $(28.5-31.3 \%)$, fine and very fine sand (26.0-26.5\%), and silt (30.0-30.6\%). The topsoil $(1-15 \mathrm{~cm})$ had a higher proportion of medium sand than in the deeper layer $(15-30 \mathrm{~cm})$. However, it contained a lower proportion of large sand particles and clay particles indicating the intensity of dolomite weathering. There were equally high contents of very fine and fine sand and silt contents in the topsoil, while their proportion was lower at the deeper soil layer.

In both layers, the soil $\mathrm{pH}$ was weakly acidic (nearly neutral), the soil was highly saturated with exchangeable cations, rich in organic matter and with high total nitrogen content. The decomposition of organic matter and its turnover in the soil was intense ( $\mathrm{C} / \mathrm{N}$ was $15-18)$; accumulation of organic matter was not detected. The soil had high concentration of $\mathrm{K}, \mathrm{Ca}, \mathrm{Mg}$. Increased concentration of $\mathrm{K}$ was found in the soil up to $15 \mathrm{~cm}$ depth where the plants have more significant role in its turnover, while the concentration of $\mathrm{Ca}$ and $\mathrm{Mg}$ was higher in the deeper layer close to the dolomite bedrock (Table 1). During the field studies, at all soil depths, the application of hydrochloric acid $10 \% \mathrm{HCl}$ caused low foam or little bubbling indicating the presence of carbonates. The soil contained a large concentration of $\mathrm{Fe}$ and $\mathrm{Mn}$ that may be related to a large amount of both elements in the bedrock.

Plant communities. The cluster analysis of 20 relevés resulted into three distinct groups that were named after the characteristic species: (1) Filipendula vulgaris-Pinus sylvestris community; (2) Lathyrus niger-Quercus robur community, and (3) Corylus avellana community. The description of the location of each sample plot, the main parameters of each vegetation layer and the covers are given in Table 2.

The DCA ordination (Fig. 2) shows a clear distinction of the above mentioned three plant community groups. The spatial pattern of the relevés in the ordination space is determined primarily by Axis 1 (eigenvalue = 0.82), with less explained variation of Axis 2 (eigenvalue $=0.20$ ). The
Table 1. Granulometric fractions, physical and chemical properties of soil along the transect Vegi1-Vegi5

\begin{tabular}{|c|c|c|c|}
\hline \multirow[t]{2}{*}{ Soil parameters } & \multirow[t]{2}{*}{ Unit } & \multicolumn{2}{|c|}{ Depth, cm } \\
\hline & & $1-15$ & $15-30$ \\
\hline$\underline{\text { Soil texture }}$ & $\mathrm{mm}, \%$ & & \\
\hline Coarse and very coarse sand & $0.63-2.00$ & $8.8 \pm 0.4$ & $9.8 \pm 0.8$ \\
\hline Medium sand & $0.20-0.63$ & $31.3 \pm 1.8$ & $28.5 \pm 1.2$ \\
\hline Fine and very fine sand & $0.063-0.200$ & $26.0 \pm 0.6$ & $26.5 \pm 1.1$ \\
\hline Silt & $0.002-0.063$ & $30.0 \pm 2.4$ & $30.6 \pm 1.8$ \\
\hline Clay & $<0.002$ & $3.9 \pm 0.3$ & $4.6 \pm 0.7$ \\
\hline \multicolumn{4}{|l|}{ Acidity, carbon and nitrogen } \\
\hline Exchangeable acidity, $\mathrm{pH}_{\mathrm{KCl}}$ & & $6.5 \pm 0.3$ & $6.8 \pm 0.1$ \\
\hline Exchangeable bases & $\mathrm{cmol} \cdot \mathrm{kg}^{-1}$ & $49.5 \pm 0.8$ & $39.8 \pm 7.7$ \\
\hline Base saturation & $\%$ & $97 \pm 1$ & $93 \pm 4$ \\
\hline Organic carbon, $\mathrm{C}_{\mathrm{org}}$ & $\mathrm{g} \cdot \mathrm{kg}^{-1}$ & $38.1 \pm 4.3$ & $27.3 \pm 7.7$ \\
\hline Total nitrogen, $\mathrm{N}_{\text {tot }}$ & $\mathrm{g} \cdot \mathrm{kg}^{-1}$ & $2.1 \pm 0.3$ & $1.4 \pm 0.2$ \\
\hline $\mathrm{C} / \mathrm{N}$ & & $18.1 \pm 0.7$ & $15.6 \pm 3.2$ \\
\hline \multicolumn{4}{|l|}{ Macroelements } \\
\hline K & $\mathrm{mg} \cdot \mathrm{kg}^{-1}$ & $192 \pm 12$ & $113 \pm 11$ \\
\hline $\mathrm{Mg}$ & $\mathrm{mg} \cdot \mathrm{kg}^{-1}$ & $5854 \pm 1470$ & $8400 \pm 3352$ \\
\hline $\mathrm{Ca}$ & $\mathrm{mg} \cdot \mathrm{kg}^{-1}$ & $12543 \pm 2875$ & $17411 \pm 6937$ \\
\hline $\mathrm{Fe}$ & $\mathrm{mg} \cdot \mathrm{kg}^{-1}$ & $1178 \pm 122$ & $1074 \pm 190$ \\
\hline $\mathrm{Mn}$ & $\mathrm{mg} \cdot \mathrm{kg}^{-1}$ & $654 \pm 121$ & $840 \pm 101$ \\
\hline
\end{tabular}

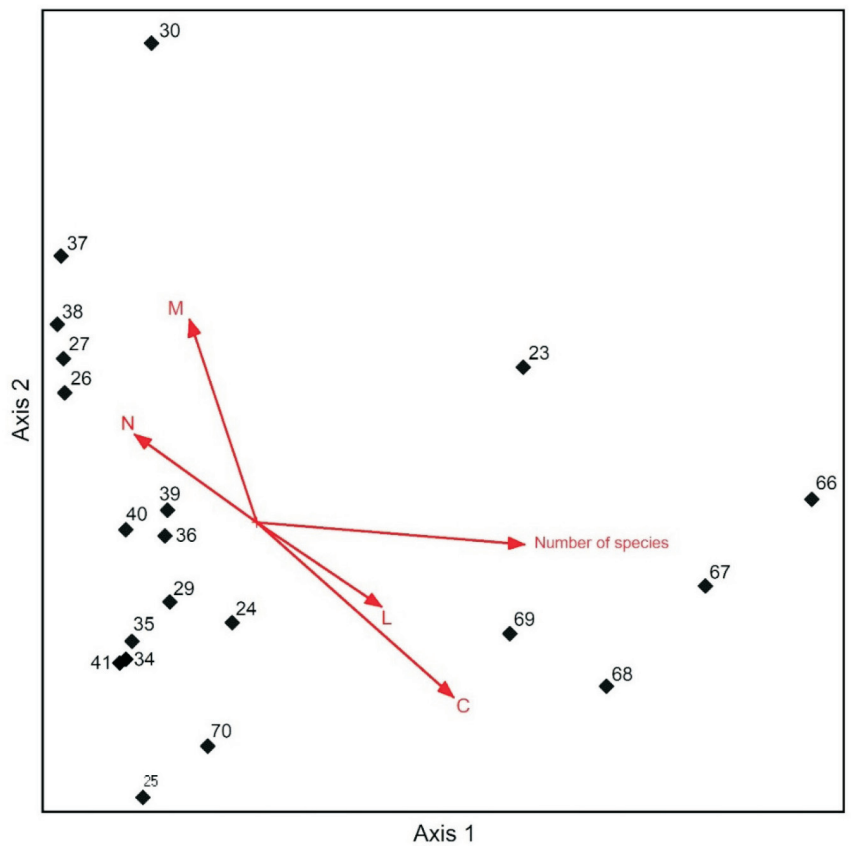

Fig. 2. DCA ordination of vegetation relevés. Abbreviations: L, light; T, temperature; C, continentality; M, moisture; N, nitrogen (Ellenberg indicator values). The numbers of relevés are given as in Table 2 .

quasi-climax oak forest relevés occur in the lower left corner, while the relevés representing Corylus avellana community - at the upper left side of the ordination graph. The relevés of young forest pine forest stage and juniper facies occur on the right side. The groups are quite well distinguishable with a poorly pronounced intermediate stage that may be related to land use history, as suggested by the tree ages (see Discussion). 
Table 2. Plant communities at the study site. The IDs of sample plots Vegi1-Vegi5 are modified to the numbers in the database, i.e., Vegi1 $=66$, Vegi2 $=67$, Vegi3 $=68$, Vegi4 $=69$, Vegi5 $=70$

\begin{tabular}{|c|c|c|c|c|c|c|c|c|c|c|c|c|c|c|c|c|c|c|c|c|c|c|c|c|}
\hline \multirow[t]{3}{*}{ Species/Syntaxa } & \multirow{4}{*}{ 离 } & \multicolumn{23}{|c|}{ Plant communities } \\
\hline & & \multicolumn{6}{|c|}{$\begin{array}{c}\begin{array}{c}\text { Filipendula vulgaris-Pinus sylvestris } \\
\text { community }\end{array} \\
\end{array}$} & \multicolumn{11}{|c|}{ Lathyrus niger-Quercus robur community } & \multicolumn{6}{|c|}{ Corylus avellana community } \\
\hline & & 1 & 2 & 3 & 4 & 5 & & 6 & 7 & 8 & 9 & 10 & 11 & 12 & 13 & 14 & 15 & & 16 & 17 & 18 & 19 & 20 & \\
\hline Relevé number in data base & & 66 & 67 & 68 & 23 & 69 & $s^{\circ}$ & 24 & 25 & 70 & 29 & 39 & 36 & 34 & 41 & 35 & 40 & 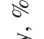 & 26 & 27 & 38 & 37 & 30 & \\
\hline Cover tree layer $(\%)$ & & 6 & 40 & 75 & 55 & 90 & ১্ট & 90 & 55 & 55 & 70 & 65 & 80 & 70 & 60 & 60 & 65 & 仓े & 0 & 5 & 0 & 0 & 0 & 毵 \\
\hline Cover shrub layer (\%) & & 5 & 20 & 10 & 15 & 5 & च & 30 & 30 & 80 & 45 & 70 & 65 & 65 & 60 & 60 & 90 & 气̆ & 85 & 90 & 95 & 95 & 90 & \\
\hline Cover herb layer (\%) & & 99 & 80 & 80 & 75 & 40 & 苞 & 65 & 65 & 40 & 55 & 70 & 35 & 75 & 70 & 70 & 50 & 总 & 70 & 55 & 40 & 20 & 65 & 离 \\
\hline Cover moss layer (\%) & & 0 & 20 & 70 & 5 & 10 & 式 & 1 & 0 & 0 & 0 & 0 & 1 & 0 & 0 & 1 & 1 & & 0 & 0 & 0 & 0 & 0 & \\
\hline Relevč area, m2 & & 314 & 314 & 314 & 350 & 314 & & 425 & 350 & 700 & 400 & 400 & 350 & 300 & 400 & 400 & 400 & & 400 & 400 & 400 & 400 & 400 & \\
\hline Aspect, degrees & & 210 & 180 & 170 & 180 & 180 & & 170 & 170 & 180 & 190 & 180 & 180 & 110 & 80 & 180 & 180 & & 90 & 150 & 180 & 180 & $\cdot$ & \\
\hline Number of species & & 42 & 39 & 43 & 52 & 41 & & 33 & 21 & 35 & 30 & 27 & 25 & 23 & 24 & 23 & 28 & & 13 & 13 & 15 & 16 & 28 & \\
\hline
\end{tabular}

Cl. Festuco-Brometea Br.-Bl et Tx. ex Soo 1947, All. Filipendulo vulgaris-Helictotrichon pratensis Dengler et Lobel in Dengler et al. 2003

$\begin{array}{lllllllll}\text { Filipendula vulgaris } & \text { E1 } & 20 & 15 & 6 & 5 & + & 100 & . \\ \text { Carex flacca } & \text { E1 } & 4 & 2 & 4 & 10 & 5 & 100 & 1 \\ \text { Helictotrichon pratense } & \text { E1 } & 25 & 8 & 6 & 3 & + & 100 & . \\ \text { Galium boreale } & \text { E1 } & 7 & 1 & 5 & 1 & + & 100 & . \\ \text { Knautia arvensis } & \text { E1 } & + & . & 2 & + & + & 80 & . \\ \text { Brachypodium pinnatum } & \text { E1 } & . & 12 & 10 & 8 & 15 & 80 & . \\ \text { Pimpinella saxifraga } & \text { E1 } & + & 3 & 1 & . & . & 60 & . \\ \text { Inula salicina } & \text { E1 } & 8 & 2 & . & + & . & 60 & . \\ \text { Briza media } & \text { E1 } & 10 & 5 & . & + & . & 60 & . \\ \text { Daucus carota } & \text { E1 } & 4 & + & . & . & . & 40 & . \\ \text { Fragaria viridis } & \text { E1 } & 3 & + & . & . & . & 40 & . \\ \text { Carlina vulgaris } & \text { E1 } & 1 & 3 & . & . & . & 40 & . \\ \text { Leontodon hispidus } & \text { E1 } & + & + & . & . & . & 40 & . \\ \text { Poa angustifolia } & \text { E1 } & + & . & . & 1 & . & 40 & . \\ \text { Plantago lanceolata } & \text { E1 } & + & . & . & + & . & 40 & . \\ \text { Cirsium acaule } & \text { E1 } & . & 5 & . & . & . & 20 & . \\ \text { Sesleria caerulea } & \text { E1 } & . & . & . & 1 & . & 20 & . \\ \text { Viola hirta } & \text { E1 } & & & & + & & 20 & .\end{array}$

\begin{tabular}{lllllllll} 
Sesleria caerulea & E1 & $\cdot$ &. & $\cdot$ & 1 &. & 20 \\
Viola hirta & E1 &. &. &. & + &. & 20 \\
\hline
\end{tabular}

Cl. Trifolio-Geranietea T. Muller 1962, All. Geranion sanguinei Tx. in T. Muller 1962

\begin{tabular}{lccccccc} 
Origanum vulgare & E1 & 12 & 4 & 3 & 2 & + & 100 \\
Agrimonia eupatoria & E1 & 6 & 18 & + & 3 & 4 & 100 \\
Calamagrostis epigeios & E1 & 7 & 3 & 2 & 8 & 1 & 100 \\
Geranium sanguineum & E1 &. & 10 & 5 & 1 & + & 80 \\
Ranunculus polyanthemos & E1 & + &. &. &. &. & 20 \\
Astragalus glycvphyllos & E1 &. & + &. &. &. & 20 \\
\hline
\end{tabular}

Cl. Quercetea pubescentis Doing-Kraft ex Scamoni et Passarge 1959, All. Quercion pubescenti-petraeae Br.-Bl. npm. mut.

\begin{tabular}{|c|c|c|c|c|c|c|c|c|c|c|c|c|c|c|c|c|c|c|c|c|c|c|c|c|}
\hline \multirow[b]{2}{*}{ Veronica teucrium } & \\
\hline & E1 & 5 & 3 & 1 & 1 & + & 100 & . & . & . & . & + & . & . & . & . & . & 10 & . & . & . & . & . & . \\
\hline Rhamnus cathartica & E2 & + & 2 & + & . & + & 80 & + & . & + & . & . & . & . & . & . & . & 20 & . & . & . & . & . & . \\
\hline Campanula persicifolia & E1 & + & . & + & + & . & 60 & + & . & . & + & + & . & . & . & + & . & 30 & . & . & . & . & + & 20 \\
\hline Clinopodium vulgare & E1 & + & 1 & . & 1 & . & 60 & . & . & . & . & . & . & . & . & . & + & 10 & . & . & . & . & . & . \\
\hline Ligustrum vulgare & E2 & 1 & 5 & . & . & . & 40 & . & . & . & . & . & . & . & . & . & . & . & . & . & . & . & . & . \\
\hline Melica nutans & E1 & . & . & . & 4 & . & 20 & 4 & + & + & . & 6 & 2 & . & + & + & . & 70 & . & . & . & . & 2 & 20 \\
\hline Crataegus curvisepala & E2 & + & . & . & . & . & 20 & . & + & 2 & . & . & . & + & + & + & . & 50 & . & . & . & . & . & . \\
\hline Rosa rubiginosa & $\begin{array}{l}\text { E2 } \\
\text { F2 }\end{array}$ & i & 1 & . & . & + & 20 & . & + & + & . & . & . & . & . & . & . & 20 & . & . & . & . & + & 20 \\
\hline \multicolumn{25}{|c|}{ Asoc. Lathyro nigri-Quercetum roboris Bulokhov et Solomechsh 2003 in Bulokhov et Solomescsh 2003} \\
\hline Viola mirabilis & E1 & . & . & . & + & 1 & 40 & 4 & 2 & 1 & 6 & 4 & 4 & + & 4 & 2 & 5 & 100 & 3 & 5 & 6 & . & + & 80 \\
\hline Lathyrus niger & E1 & . & . & 8 & 1 & 1 & 60 & 4 & + & 2 & + & 8 & 2 & 1 & + & 3 & 2 & 100 & . & . & . & . & 3 & 20 \\
\hline Primula veris & E1 & 3 & 8 & 5 & 6 & 2 & 100 & 4 & . & + & 8 & 4 & 6 & . & . & . & 2 & 60 & . & . & . & . & + & 20 \\
\hline Convallaria majalis & E1 & . & . & . & . & . & . & 8 & 35 & 25 & . & . & . & 25 & 20 & 25 & 3 & 60 & . & . & . & . & . & . \\
\hline Carex montana & E1 & . & . & . & . & . & . & + & . & + & . & . & . & . & . & . & . & 20 & . & . & . & . & . & . \\
\hline Anemone nemorosa & E1 & . & . & . & 2 & . & 20 & 10 & 20 & 3 & 10 & . & + & 10 & 7 & 8 & 8 & 90 & 20 & 40 & 10 & 5 & . & 80 \\
\hline Viburnum opulus & E2 & . & + & . & . & + & 40 & + & . & 1 & . & + & + & + & + & + & + & 80 & . & + & + & + & + & 80 \\
\hline Tilia cordata & E2 & . & . & . & . & . & . & . & . & . & 3 & 5 & 2 & + & 5 & . & + & 60 & + & + & + & . & . & 60 \\
\hline Paris quadrifolia & E1 & . & . & . & . & . & . & 3 & + & + & + & + & . & . & . & . & + & 60 & 2 & + & + & + & + & 100 \\
\hline Aegopodium podagraria & E1 & . & . & . & 15 & 1 & 40 & 6 & . & . & + & 5 & 3 & . & + & . & + & 60 & . & . & . & . & 20 & 20 \\
\hline Carex digitata & E1 & . & . & . & . & + & 20 & + & . & + & + & . & + & . & . & + & + & 60 & . & . & . & . & . & . \\
\hline Euonymus europaea & E2 & . & . & + & . & . & 20 & . & . & . & + & + & + & + & 1 & . & . & 50 & . & . & . & + & . & 20 \\
\hline Malus sylvestris & E3 & . & . & . & . & 5 & 20 & . & . & . & . & . & . & . & . & . & . & . & . & . & . & . & . & . \\
\hline Malus sylvestris & E2 & . & . & . & + & . & 20 & + & + & . & + & + & . & . & + & . & . & 50 & . & . & + & . & . & 20 \\
\hline Ranunculus cassubicus & E1 & . & . & . & . & . & . & 1 & . & . & . & . & + & + & . & + & 1 & 50 & + & . & + & . & . & 40 \\
\hline Brachypodium sylvaticum & E1 & . & . & . & . & . & . & . & . & . & + & 8 & 4 & . & . & 1 & . & 40 & . & . & . & 1 & 2 & 40 \\
\hline Galeobdolon luteum & E1 & . & . & . & . & . & . & . & . & . & . & . & . & . & . & 6 & . & 10 & . & . & . & . & 8 & 20 \\
\hline Geum urbanum & E1 & . & . & . & . & . & . & . & . & . & . & . & . & . & + & . & . & 10 & + & + & + & + & + & 100 \\
\hline Daphne mezereum & E2 & . & . & . & . & . & . & . & . & + & . & . & . & . & . & . & . & 10 & . & . & + & . & . & 20 \\
\hline Poa nemoralis & E1 & . & . & . & . & . & . & . & . & . & . & 4 & . & . & . & . & . & 10 & . & . & . & . & . & . \\
\hline Lathyrus vernus & E1 & . & . & . & . & . & . & . & . & + & . & . & . & . & . & . & . & 10 & . & . & . & . & . & . \\
\hline Actaea spicata & E1 & . & . & . & . & . & . & . & . & . & . & . & . & . & . & . & . & 10 & . & . & . & . & . & \\
\hline Scrophularia nodosa & E1 & & & & & & & & & & & & + & & & & & 10 & & & & & & \\
\hline
\end{tabular}


Table 2 (continued)

\begin{tabular}{|c|c|c|c|c|c|c|c|c|c|c|c|c|c|c|c|c|c|c|c|c|c|c|c|c|}
\hline \multirow[t]{2}{*}{ Species/Syntaxa } & \multirow{11}{*}{ } & \multicolumn{23}{|c|}{ Plant communities } \\
\hline & & \multicolumn{6}{|c|}{$\begin{array}{c}\text { Filipendula vulgaris-Pinus sylvestris } \\
\text { community }\end{array}$} & \multicolumn{11}{|c|}{ Lathyrus niger-Quercus robur community } & & rylus & avella & na cor & nmuni & \\
\hline Relevé number & & 1 & 2 & 3 & 4 & 5 & & 6 & 7 & 8 & 9 & 10 & 11 & 12 & 13 & 14 & 15 & & 16 & 17 & 18 & 19 & 20 & \\
\hline Relevé number in data base & & 66 & 67 & 68 & 23 & 69 & & 24 & 25 & 70 & 29 & 39 & 36 & 34 & 41 & 35 & 40 & & 26 & 27 & 38 & 37 & 30 & \& \\
\hline Cover tree layer $(\%)$ & & 6 & 40 & 75 & 55 & 90 & $\delta^{\circ}$ & 90 & 55 & 55 & 70 & 65 & 80 & 70 & 60 & 60 & 65 & $\therefore$ & 0 & 5 & 0 & 0 & 0 & 3 \\
\hline Cover shrub layer $(\%)$ & & 5 & 20 & 10 & 15 & 5 & ১̀ & 30 & 30 & 80 & 45 & 70 & 65 & 65 & 60 & 60 & 90 & 产 & 85 & 90 & 95 & 95 & 90 & ల్ \\
\hline Cover herb layer (\%) & & 99 & 80 & 80 & 75 & 40 & $\underset{D}{\vec{D}}$ & 65 & 65 & 40 & 55 & 70 & 35 & 75 & 70 & 70 & 50 & $\overline{\bar{g}}$ & 70 & 55 & 40 & 20 & 65 & 콜 \\
\hline Cover moss layer (\%) & & 0 & 20 & 70 & 5 & 10 & छ్ّ & 1 & 0 & 0 & 0 & 0 & 1 & 0 & 0 & 1 & 1 & 总 & 0 & 0 & 0 & 0 & 0 & 党 \\
\hline Relevč area, m2 & & 314 & 314 & 314 & 350 & 314 & & 425 & 350 & 700 & 400 & 400 & 350 & 300 & 400 & 400 & 400 & & 400 & 400 & 400 & 400 & 400 & \\
\hline Aspect, degrees & & 210 & 180 & 170 & 180 & 180 & & 170 & 170 & 180 & 190 & 180 & 180 & 110 & 80 & 180 & 180 & & 90 & 150 & 180 & 180 & . & \\
\hline Number of species & & 42 & 39 & 43 & 52 & 41 & & 33 & 21 & 35 & 30 & 27 & 25 & 23 & 24 & 23 & 28 & & 13 & 13 & 15 & 16 & 28 & \\
\hline Polygonatum multiflorum & E1 & . & . & . & . & . & . & . & . & . & . & . & . & . & . & . & . & . & 1 & . & . & . & . & 20 \\
\hline Lonicera xylosteum & E2 & . & . & . & . & . & . & . & . & . & . & . & . & . & . & . & . & . & . & . & . & + & . & 20 \\
\hline Pulmonaria obscura & E1 & . & . & . & . & . & . & . & . & . & . & . & . & . & . & . & . & . & . & . & . & . & 4 & 20 \\
\hline Cerasus avium & E2 & . & . & . & . & . & . & . & . & . & . & . & . & . & . & . & . & . & . & . & . & . & 2 & 20 \\
\hline Asarum europaeum & E2 & & & & & & & & & & & & & & & & & & & & & & 1 & 20 \\
\hline & & & & & & & & & & her $\mathrm{sp}$ & ecies & & & & & & & & & & & & & \\
\hline Pinus sylvestris & E3 & 6 & 40 & 60 & 20 & 40 & 100 & + & . & 5 & . & . & . & 5 & + & . & . & 40 & . & . & . & . & . & . \\
\hline Pinus sylvestris & E2 & 2 & + & . & . & . & 40 & . & . & . & . & . & . & . & . & . & . & . & . & . & . & . & . & . \\
\hline Pinus sylvestris & E1 & . & + & . & . & . & 20 & . & . & . & . & . & . & . & . & . & . & . & . & . & . & . & . & . \\
\hline Juniperus communis & E2 & 3 & 15 & 6 & 4 & + & 100 & 2 & . & . & . & . & . & . & . & . & . & 10 & . & . & . & . & . & \\
\hline Juniperus communis & E3 & . & + & 1 & . & . & 40 & . & . & . & . & . & . & . & . & . & . & . & . & . & . & . & . & . \\
\hline Quercus robur & E3 & + & . & . & . & 15 & 40 & 80 & 55 & 45 & 65 & 65 & 80 & 65 & 60 & 60 & 65 & 100 & . & . & + & . & . & 20 \\
\hline Quercus robur & E2 & + & . & 1 & 2 & 1 & 80 & . & . & . & . & . & . & . & . & . & . & . & . & . & . & . & + & 20 \\
\hline Quercus robur & E1 & . & + & + & 2 & + & 80 & . & . & + & . & . & . & . & . & . & . & 10 & . & . & + & . & . & 20 \\
\hline Corylus avellana & E2 & . & . & + & 8 & 3 & 60 & 25 & 25 & 25 & 35 & 60 & 60 & 65 & 50 & 55 & 65 & 100 & 85 & 90 & 90 & 95 & 85 & 100 \\
\hline Sorbus aucuparia & E2 & . & + & + & + & 2 & 80 & . & + & + & 5 & 3 & 1 & + & + & + & + & 90 & . & . & + & . & 1 & 40 \\
\hline Padus avium & E2 & . & . & . & . & + & 20 & . & 3 & 10 & + & . & . & + & . & . & . & 40 & 10 & 15 & 5 & + & 5 & 100 \\
\hline Picea abies & E2 & . & . & . & 2 & + & 40 & 2 & 2 & + & + & + & . & + & + & . & + & 80 & . & . & . & . & . & . \\
\hline Picea abies & E3 & . & . & 15 & . & + & 20 & + & 3 & 10 & . & . & . & . & . & . & + & 40 & . & . & . & . & . & . \\
\hline Populus tremula & E3 & . & . & . & 30 & 30 & 40 & 10 & + & 5 & . & . & . & . & . & . & . & 30 & . & . & . & . & . & . \\
\hline Populus tremula & E2 & . & . & . & 1 & 1 & 40 & . & . & + & . & . & . & . & . & . & . & 10 & . & . & . & . & . & $\cdot$ \\
\hline Populus tremula & E1 & . & . & . & . & 2 & 20 & . & . & + & . & . & . & . & . & . & . & 10 & . & . & . & . & . & \\
\hline Fraxinus excelsior & E2 & . & . & + & . & + & 40 & . & . & . & . & + & . & . & . & + & . & 20 & . & . & . & . & . & . \\
\hline Acer platanoides & E2 & . & . & + & . & . & 20 & . & . & + & + & . & . & + & 1 & + & + & 60 & . & + & . & . & . & 20 \\
\hline Acer platanoides & E1 & . & . & . & + & . & 20 & . & . & + & . & . & . & . & . & . & + & 20 & . & . & . & . & . & . \\
\hline Betula pendula & E3 & . & . & . & 5 & . & 20 & . & . & . & . & . & . & . & . & . & . & . & . & . & . & . & . & . \\
\hline Frangula alnus & E2 & . & + & . & + & . & 40 & . & . & . & . & . & . & . & . & . & . & . & . & . & . & . & . & . \\
\hline Ulmus glabra & E2 & . & . & . & . & . & . & . & . & . & . & . & . & + & . & . & . & 10 & . & . & . & . & + & 20 \\
\hline Ulmus glabra & E1 & . & . & + & . & + & 40 & . & . & + & . & . & . & . & . & . & . & 10 & . & . & . & . & . & . \\
\hline Geranium sylvaticum & E1 & . & . & . & . & . & . & 1 & + & 1 & . & 3 & 1 & 2 & 5 & 1 & 2 & 90 & . & . & + & . & 3 & 40 \\
\hline Chaerophyllum aromaticum & E1 & . & . & . & . & . & . & 2 & . & . & + & 4 & . & + & . & + & 1 & 60 & . & . & . & 1 & + & 40 \\
\hline Calamagrostis arundinacea & E1 & . & . & . & . & . & . & . & 2 & 1 & . & . & . & . & . & 3 & + & 40 & . & . & . & . & . & . \\
\hline Rubus saxatilis & E1 & . & . & . & . & . & . & + & . & + & . & . & . & + & + & . & . & 40 & . & . & . & . & . & - \\
\hline Maianthemum bifolium & E1 & . & . & . & . & . & 20 & . & + & . & 1 & . & . & + & . & . & + & 40 & . & . & . & . & . & . \\
\hline Ranunculus acris & E1 & . & . & . & + & . & 20 & . & . & . & + & + & + & . & . & . & + & 40 & . & . & . & . & . & . \\
\hline Pteridium aquilinum & E1 & . & . & . & 2 & . & 20 & 2 & . & . & + & . & + & . & + & . & . & 40 & . & . & . & + & . & 20 \\
\hline Melampyrum nemorosum & E1 & . & . & . & 3 & . & 20 & 3 & . & . & 6 & . & 3 & . & . & . & . & 30 & . & . & . & . & 15 & 20 \\
\hline Taraxacum officinale & E1 & . & . & . & + & . & 20 & . & . & + & + & . & . & . & . & . & . & 20 & . & . & . & + & + & 40 \\
\hline Luzula pilosa & E1 & . & . & . & + & . & 20 & + & . & . & . & . & . & . & . & . & + & 20 & . & . & . & . & . & . \\
\hline Solidago virgaurea & E1 & . & . & 1 & . & + & 40 & . & . & + & . & + & . & . & . & . & . & 20 & . & . & . & . & . & . \\
\hline Veronica chamaedrys & E1 & . & . & . & 2 & + & 40 & . & . & . & + & . & . & . & . & . & . & 10 & . & . & . & . & + & 20 \\
\hline Rhytidiadelphus triquetrus & E0 & . & 10 & 20 & 5 & 10 & 80 & 1 & . & . & . & . & . & . & . & . & . & 10 & . & . & . & . & . & . \\
\hline Fragaria vesca & E1 & . & . & 2 & 3 & 3 & 60 & . & . & . & . & . & . & . & . & . & . & $\cdot$ & . & . & . & . & . & . \\
\hline Galium album & E1 & 2 & + & 1 & 3 & 1 & 100 & . & . & . & . & . & . & . & . & . & . & . & . & . & . & . & . & . \\
\hline Dactylis glomerata & E1 & + & + & + & 1 & + & 100 & . & . & . & . & . & . & . & . & . & . & . & . & . & . & . & . & . \\
\hline Leucanthemum vulgare & E1 & + & 1 & + & . & . & 60 & . & . & . & . & . & . & . & . & . & . & . & . & . & . & . & . & . \\
\hline Vicia cracca & E1 & 1 & + & + & . & . & 60 & . & . & . & . & . & . & . & . & . & . & . & . & . & . & . & . & . \\
\hline Scorzonera humilis & E1 & . & . & + & . & 1 & 40 & . & . & . & . & . & . & . & . & . & . & . & . & . & . & . & . & . \\
\hline Brachythecium albicans & E0 & . & 10 & 1 & . & . & 40 & . & . & . & . & . & . & . & . & . & . & . & . & . & . & . & . & . \\
\hline Scleropodium purum & E0 & . & . & 50 & . & + & 40 & . & . & . & . & . & . & . & . & . & . & . & . & . & . & . & . & . \\
\hline Eurhynchium hians & E0 & . & . & . & . & . & . & . & . & . & . & . & . & . & . & 1 & 1 & 20 & . & . & . & . & . & . \\
\hline Fragaria moschata & E1 & . & . & . & . & . & . & 1 & . & . & . & . & . & . & . & . & . & 10 & + & + & . & . & . & 40 \\
\hline
\end{tabular}

Rare species:

Tree layer: Alnus incana 5(17); shrub layer: Cerasus vulgaris 4(3), Ribes spicatum $+(8),+(9)$, Alnus incana $+(17),+(19)$; herb layer: Convolvulus arvensis +(1), 1(2), Lathyrus pratensis +(1), Centaurea jacea 2(1), Medicago lupulina 2(1), Silene vulgaris 1(1), Senecio jacobaea $+(1)$, Phleum pratense $+(1)$, Potentilla reptans $+(1)$, Prunella vulgaris $+(2)$, Agrostis tenuis $+(2)$, Melampyrum polonicum $1(3),+(5)$, Leontodon autumnalis $+(3)$, Ophioglossum vulgatum 6(4), Vicia sepium 1(4), Festuca pratensis $+(4)$, Helictotrichon pubescens $+(4)$, Ononis arvensis $+(4)$, Carex hirta $+(6)$, Rubus idaeus $+(7),+(20)$, Hieracium vulgatum $+(8)$, Carex contigua $+(8)$, Rubus caesius $+(9)$, Deschampsia cespitosa $+(10),+(15)$, Melandrium dioicum $+(10)$, Carex vaginata $+(11)$, Stellaria media $+(11)$, Anemone ranunculoides 5(16), Allium oleraceum 1(19). 
Among the environmental variables used in the ordination, continentality, moisture and nitrogen appeared to be the most significant (the tightest correlation with the axes), while others were of lower importance (Table 3). The young forest stage with pines and junipers had the highest proportion of light demanding continental and subcontinental grassland and forest edge species, while mature forest community was composed of shade tolerant species. Not surprisingly, the moisture and nutrient levels were higher in the mature forest stage than in the young forest stage. The number of species was higher in the young forest stage that follows semi-open xeric grassland (see also Table 2).

The number of character species of various phytosociological classes varied at different succession stages (Fig. 3).

The succession of semi-natural xeric calcareous grassland toward formation of mature oak forest was represented by five sample plots located in a transect across a representative area. The first three sample plots (Vegi1-Vegi3) represented young overgrowing stages with pine at the age of 10 to 25 years, while Vegi5 represented the quasi-climax succession stage with more than 200-year-old oak forest. The 40-year-old pine stand (Vegi4) represented the transition from medium-aged pine forest at the oak forest edge to mature oak forest.

At the youngest forest stage, 10- to 15-year-old pine stand, species of Festuco-Brometea grasslands dominated. In the 25-year-old pine stand the number of Festuco-Brometea species decreased by 2.3 times. The most resistant grassland species during the pine and juniper encroachment are Filipendula vulgaris, Helictotrichon pratensis and Carex flacca (frequency 60\%). In the 40-year-old stand, there was high

Table 3. Kendall correlation coefficients of the DCA axes with the means of Ellenberg indicator values. Correlation coefficients $>0.50$ are given in bold

\begin{tabular}{lccc}
\hline \multicolumn{1}{c|}{ Variables } & DCA Axis 1 & DCA Axis 2 \\
\hline Light (L) & $\mathbf{0 . 6 0 7}$ & -0.301 \\
T (temperature) & 0.127 & -0.434 \\
C (continentality) & $\mathbf{0 . 7 5 8}$ & -0.379 \\
M (moisture) & -0.340 & $\mathbf{0 . 6 8 1}$ \\
R (soil pH) & -0.358 & -0.021 \\
N (nitrogen) & $-\mathbf{0 . 5 5 4}$ & 0.354 \\
Number of species & $\mathbf{0 . 7 5 9}$ & -0.122
\end{tabular}

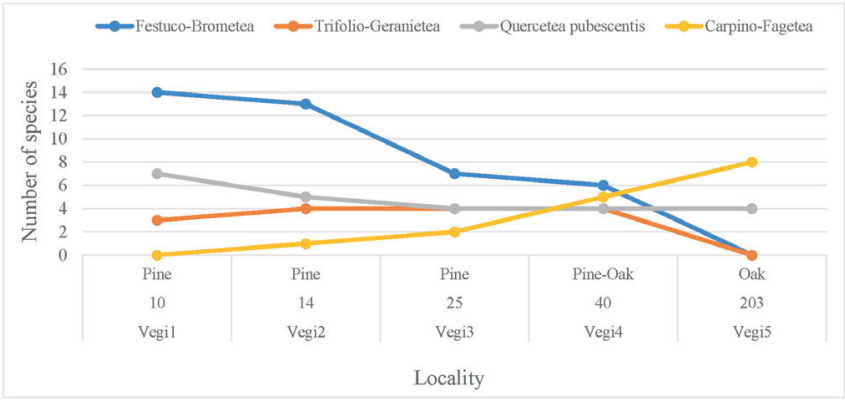

Fig. 3. Number of diagnostic species of phytosociological classes at various succession stages in the sample plots Vegi1-Vegi5. occurrence of Trifolio-Geranietea species, from which Geranium sanguineum, Origanum vulgare, Agrimonia eupatoria and Calamagrostis epigeios were the most frequent (frequency $60 \%$ ). In the mature oak forest, the species of Festuco-Brometea were absent.

The number of character species of thermophilous oak forests (Quercetea pubescentis) and suboceanic and subcontinental thermophilous scrub (the early stages of thermophilous oak forests) at various age of pine stands and the mature oak forest was relatively constant and, within the distinguished succession stages, varied from 4 to 7 species. The number of species in the overgrowing grassland (young pine stand) and forest fringe communities was similar. The most frequent species (frequency $>60 \%$ ) were Rhamnus catharica, Veronica teucrium and Campanula persicifolia.

At the same time, there was a reverse pattern of the character species of the class Carpino-Fagetea sylvaticae - they are almost absent in the young, 10-year-old and 14-year-old pine stands (Vegi1, where only one species (Viburnum opulus) was found, and Vegi2). Only two species of CarpinoFagetea sylvaticae (Hepatica nobilis and Euonymus europaeus) were found in the 25-year-old stand (Vegi3). The most frequent diagnostic species of the class CarpinoFagetea sylvaticae were Mercurialis perennis, Hepatica nobilis, Anemone nemorosa and Tilia cordata (frequency > $60 \%)$.

In the 40-year-old pine stand with an admixture of oaks (Vegi4), the number of diagnostic species of each represented phytosociological class varied from 4 to 6 . This means that in the transition zone between the young pine forest and mature oak forest all vegetation classes found in the study site were equally represented (xeric calcareous grassland, thermophilous forest fringe, thermophilous oak forest, and mesic hornbeam-beech forest).

Remarkably, in the transition zone, in the pine-oak stand (Vegi4), there was a high admixture of Populus tremula, a species that is more suitable for a local climate with a high continentality degree, rather than widespread woody pioneer species of mesic conditions, such as Betula pendula and Alnus incana.

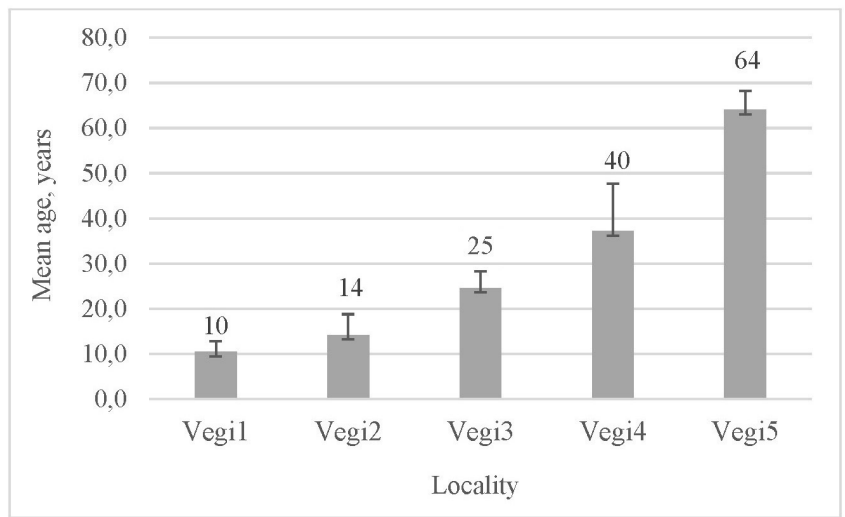

Fig. 4. Mean age of pine (with standard deviation) at various succession stages in the sample plots Vegil-Vegi5. 
Table 4. Parameters characterising each forest succession stage (sample plots Vegi1-Vegi5)

\begin{tabular}{|c|c|c|c|c|c|c|}
\hline \multirow[t]{2}{*}{ Woody species } & \multirow[t]{2}{*}{ Parameters } & \multicolumn{5}{|c|}{ Sample plots } \\
\hline & & Vegi1 & Vegi2 & Vegi3 & Vegi4 & Vegi5 \\
\hline \multirow[t]{6}{*}{ Pinus sylvestris } & Ind/ha & 701 & 540 & 1210 & 287 & 28 \\
\hline & Age, year & $10 \pm 1$ & $14 \pm 1$ & $25 \pm 1$ & $40 \pm 3$ & $64 \pm 3$ \\
\hline & $\mathrm{D}, \mathrm{cm} \_$mean & $9.1 \pm 0.7$ & $10.7 \pm 1.2$ & $13.0 \pm 1.2$ & $31.0 \pm 3.4$ & $26.4 \pm 6.1$ \\
\hline & $\mathrm{H}, \mathrm{m} \_$mean & $5.0 \pm 0.3$ & $7.0 \pm 0.5$ & $11.7 \pm 0.7$ & $18.8 \pm 1.5$ & $18.6 \pm 4.5$ \\
\hline & $\mathrm{S}, \mathrm{m}^{2} / \mathrm{ha}$ & 5.2 & 5.9 & 20.9 & 23.6 & 1.6 \\
\hline & $\mathrm{V}, \mathrm{m}^{3} / \mathrm{ha}$ & 17.6 & 26.7 & 152.9 & 223.4 & 15.6 \\
\hline \multirow[t]{6}{*}{ Juniperus communis } & Ind/ha & & 414 & 318 & & \\
\hline & Age, year & & $24 \pm 3$ & $25 \pm 2$ & & \\
\hline & $\mathrm{D}, \mathrm{cm} \_$mean & & $4.90 \pm 0.5$ & $5.7 \pm 0.6$ & & \\
\hline & $\mathrm{H}, \mathrm{m} \_$mean & & $4.2 \pm 0.3$ & $4.7 \pm 0.1$ & & \\
\hline & $\mathrm{S}, \mathrm{m}^{2} / \mathrm{ha}$ & & 0.9 & 0.9 & & \\
\hline & $\mathrm{V}, \mathrm{m}^{3} / \mathrm{ha}$ & & 3.1 & 3.1 & & \\
\hline \multirow[t]{6}{*}{ Quercus robur } & Ind/ha & 32 & & & 191 & 170 \\
\hline & Age, year & 29 & & & $30 \pm 2$ & $203 \pm 6$ \\
\hline & $\mathrm{D}, \mathrm{cm} \_$mean & 10.1 & & & $8.5 \pm 1.5$ & $53.3 \pm 5.6$ \\
\hline & $\mathrm{H}, \mathrm{m} \_$mean & 4.6 & & & $8.1 \pm 1.2$ & $21.9 \pm 1.5$ \\
\hline & $\mathrm{S}, \mathrm{m}^{2} / \mathrm{ha}$ & 0.3 & & & 1.3 & 42.5 \\
\hline & $\mathrm{V}, \mathrm{m}^{3} / \mathrm{ha}$ & 0.9 & & & 7.1 & 493.1 \\
\hline \multirow[t]{6}{*}{ Picea abies } & Ind/ha & & & 159 & 64 & 99 \\
\hline & Age, year & & & $20 \pm 1$ & 19 & $65 \pm 9$ \\
\hline & $\mathrm{D}, \mathrm{cm} \_$mean & & & $9.6 \pm 2.2$ & 5.2 & $12.3 \pm 3.2$ \\
\hline & H, m_mean & & & $8.7 \pm 1.7$ & 4.6 & $10.2 \pm 2.6$ \\
\hline & $\mathrm{S}, \mathrm{m}^{2} / \mathrm{ha}$ & & & 1.3 & 0.1 & 1.7 \\
\hline & $\mathrm{V}, \mathrm{m}^{3} / \mathrm{ha}$ & & & 7.6 & 0.3 & 13.6 \\
\hline \multirow[t]{6}{*}{ Populus tremula } & Ind/ha & & & & 541 & 14 \\
\hline & Age, year & & & & $25 \pm 1$ & 40 \\
\hline & $\mathrm{D}, \mathrm{cm} \_$mean & & & & $6.9 \pm 0.4$ & $12.1 \pm 2.9$ \\
\hline & $\mathrm{H}, \mathrm{m} \_$mean & & & & $9.3 \pm 0.6$ & $15.3 \pm 4.2$ \\
\hline & $\mathrm{S}, \mathrm{m}^{2} / \mathrm{ha}$ & & & & 2.1 & 0.2 \\
\hline & $\mathrm{V}, \mathrm{m}^{3} / \mathrm{ha}$ & & & & 11.2 & 1.2 \\
\hline \multirow[t]{6}{*}{ Malus sylvestris } & Ind/ha & & & & 159 & \\
\hline & Age, year & & & & $17 \pm 1$ & \\
\hline & $\mathrm{D}, \mathrm{cm} \_$mean & & & & $5.7 \pm 0.6$ & \\
\hline & H, m_mean & & & & $5.5 \pm 0.9$ & \\
\hline & $\mathrm{S}, \mathrm{m}^{2} / \mathrm{ha}$ & & & & 0.4 & \\
\hline & $\mathrm{V}, \mathrm{m}^{3} / \mathrm{ha}$ & & & & 1.4 & \\
\hline \multirow[t]{6}{*}{ Sorbus aucuparia } & Ind/ha & & & & 64 & \\
\hline & Age, year & & & & 21 & \\
\hline & $\mathrm{D}, \mathrm{cm} \_$mean & & & & $9.1 \pm 0.2$ & \\
\hline & $\mathrm{H}, \mathrm{m} \_$mean & & & & $9.6 \pm 0.3$ & \\
\hline & $\mathrm{S}, \mathrm{m}^{2} / \mathrm{ha}$ & & & & 0.4 & \\
\hline & $\mathrm{V}, \mathrm{m}^{3} / \mathrm{ha}$ & & & & 2.1 & \\
\hline
\end{tabular}

Tree parameters. Four sample plots within the transect (Vegi1-Vegi4) represent Pinus sylvestris stands of various age - from 10-year-old overgrowing patchy xeric grassland to medium-aged, 40- to 45-year-old mixed pine-juniper stand (Vegi4) that borders with the mature oak forest (Vegi5) (Table 4, Fig. 4) where the age of pine reached 64 years (Fig. 4). The age of junipers was ca. 25 years (Vegi2, Vegi3). The age of oak varied from 29 in the young forest (Vegi1) to 203 in the mature forest (Vegi5).
The most intense growth of pine was detected in the youngest, 10- to 15-year-old stands (Vegi1, Vegi2), where the mean annual radial increment during the last ten years (2011-2020) varied from $3.8 \mathrm{~mm}$ (Vegi1) to $2.9 \mathrm{~mm}$ (Vegi2) $(p<0.05)$ (Fig. 5). The lowest radial increment was found in the 25-year-old stand, where it was $1.3 \mathrm{~mm}$ on average, which may be explained by the large tree density (1210 individuals per ha) and, consequently, high competition among the individuals. The radial increment in Vegi1 


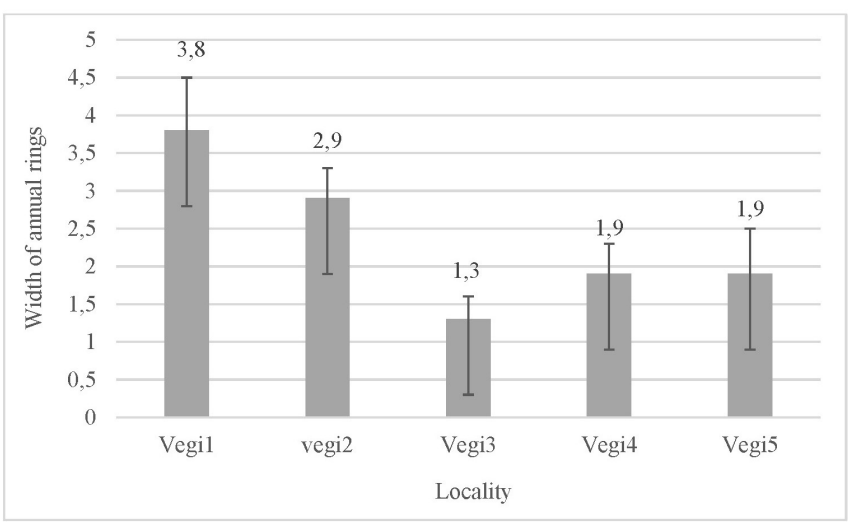

Fig. 5. Mean radial increment of pines (with standard deviation) during the last 10 years (2011-2020), in the sample plots Vegi1-Vegi5.

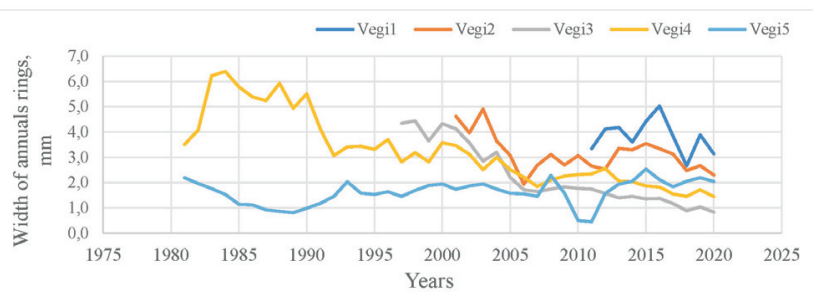

Fig. 6. Dynamics of the radial increment of pine in the sample plots Vegi1-Vegi5.

and Vegi2 was significantly lower $(p<0.01)$ than the radial increment in the neighbouring sample plots. Mean annual radial increment of the medium-aged pine in Vegi4 and Vegi5 plots during the last te years was lower than $2 \mathrm{~mm}$ and did not significantly differ within the relevant tree age class $(p=0.48)$ (Fig. 5).

The radial increment in young to medium-aged pine stands varied over years (Fig. 6). During the last years, in all sample plots a trend toward slower growth was observed, espe-

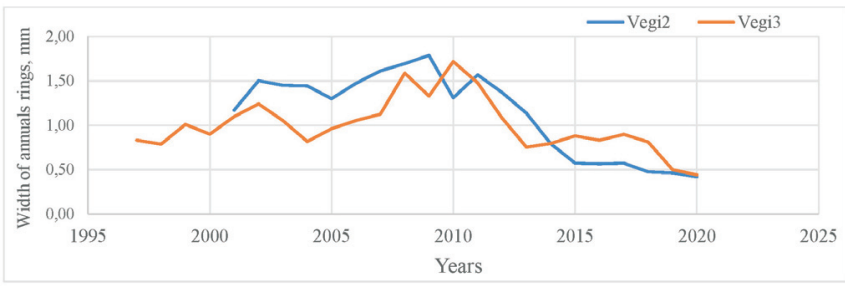

Fig. 7. Dynamics of radial increment of juniper in the sample plots Vegi2 and Vegi3.

cially in Vegi3, a pine stand with the highest tree density among the studied plots.

Individual pines at various succession stages of the formerly open grassland had different age; they differed in diameter and height, and their spatial pattern was random (Table 5). This means that, since the cessation of regular grassland management, their establishment and survival were driven by various factors. The differentiation of pine individuals by diameter and height usually occurs during the first years of tree colonisation in grasslands.

Also, overgrowing of the xeric grasslands with Juniperus communis takes place in the study site (recorded in Vegi2, Vegi3). Like pines, junipers had a random spatial pattern, rarely forming small clusters (as in Vegi3) (Table 5). In Vegi2, juniper trees were on average ten years older than pine trees (Table 4). The age of juniper varied from 17 to 37 years, while the age of pine varied from 8 to 25 years. During the last 20 years, the radial increment of juniper was synchronous $(p=0.138)$. Over the last decade, the average annual ring width of juniper in Vegi2 was $0.79 \mathrm{~mm}$, and in Vegi3 it reached $0.85 \mathrm{~mm}$ (Fig. 7).

A few oak individuals were found in the overgrowing xeric grassland with the secondary pine forest, but the largest pro-

Table 5. Spatial pattern of trees in the sample plots Vegi1-Vegi5

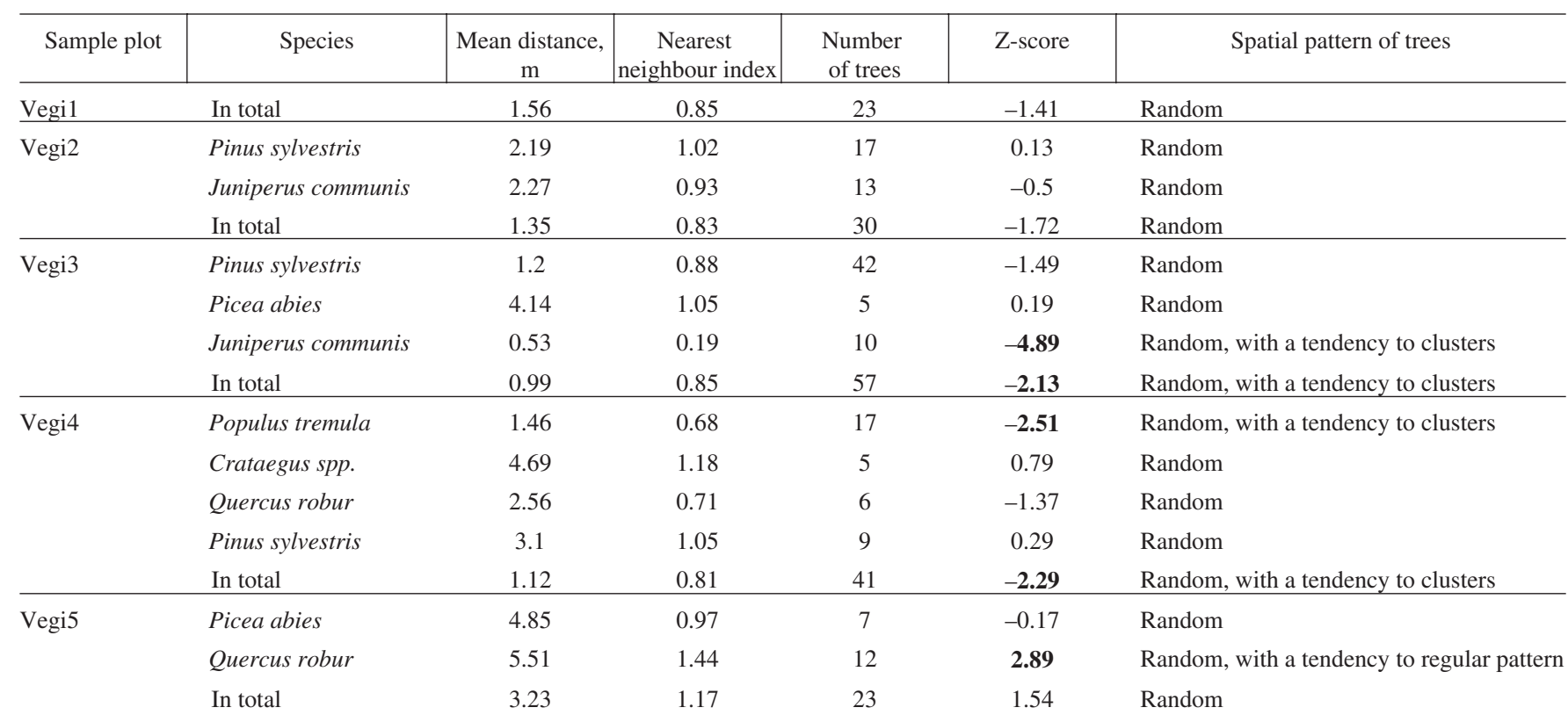

Statistically significant values are marked in bold. 


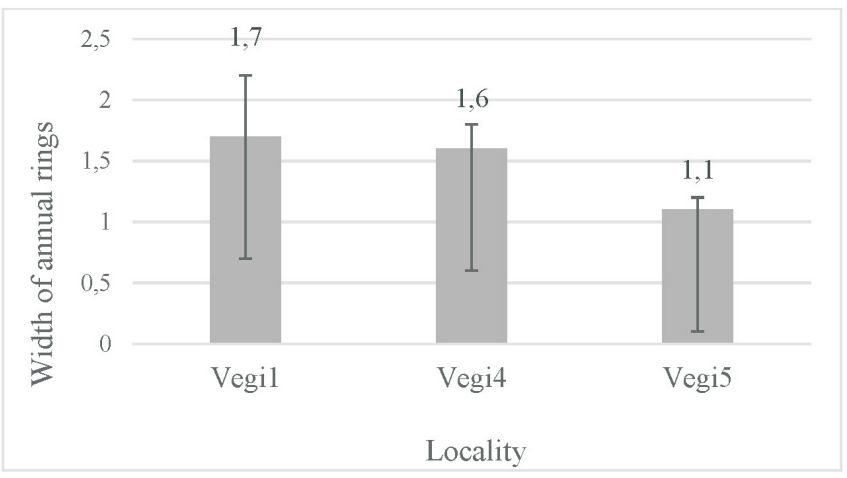

Fig. 8. Mean radial increment of oak (with standard deviation) during the last decade (2011-2020) in sample plots Veg1, Vegi4, and Vegi5.

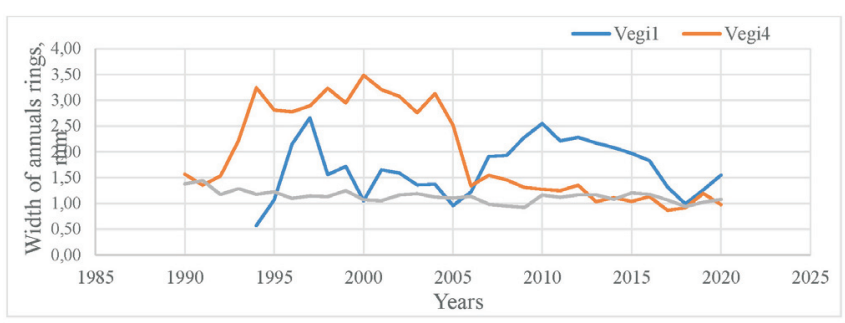

Fig. 9. Dynamics of radial increment of oak in Vegil and Vegi4.

portion of them was recorded in the area adjacent to the mature oak forest (Vegi4). The radial increment of oak in the young to medium-aged pine forest varied from 1.6 to $1.7 \mathrm{~mm}$ annually. In the mature forest the oak increment was lower (1.1 mm annually) and significantly different ( $p=0.001)$ from that of the oak individuals in the young to medium-aged pine forest (Fig. 8).

The spatial pattern of oak individuals in the entire study area was random. Regardless of the presence of dead oak trees in the stand (volume of dead oak wood was $15.2 \mathrm{~m}^{3} \cdot \mathrm{ha}^{-1}$ ) and openings in the canopy, oak tended to have a regular spatial pattern (Table 5).

During the last decades, the radial increment of oak, to some extent also pine, has stabilised in the quasi-climax oak community (Fig. 9). The radial increment of pine and other woody species in the dynamic young succession stages was highly variable (Figs. 6, 7).

\section{DISCUSSION}

According to the literature (Mucina et al., 1993; Rūsina, 2003; 2007; Wilner and Grabherr, 2007; Chytrý and Tichý, 2008; Chytrý, 2013), the character species of the following phytosociological classes and affiliated alliances, earlier recorded in Latvia, were identified in the Vegi study area: (1) class Festuco-Brometea: association Filipendulo vulgarisHelictotrichon pratensis; (2) class Trifolio-Geranietea sanguinei, alliance Geranion sanguinei; and (3) class Quercetea pubescentis: alliance Quercion pubescentipetraea, associations Carpino-Fagetea sylvaticae and Quercus robori-Tilion cordatae (nomenclature of the higher syntaxa follows Mucina et al. (2016)).
As stated by Rūsiņa (2007) and Szygendowski and Brzeg (2015), the communities of Festuco-Brometea class have a dynamic connection with the plant communities of warm, dry forest fringes. Similar to a study from Poland (Szygendowski and Brzeg, 2015), in Veǵi in the later succession stage, the character species of xeric grasslands were accompanied by species of dry, warm forest fringes, such as Geranium sanguineum, Agrimonia eupatoria, Origanum vulgare and Calamagrostis epigeios. During the overgrowing of the grassland, encroachment of woody species of CrataegusPrunetea, a scrub community of dry, warm sites, takes place. Here, the character species of scrub communities were woody species Crataegus curvisepala, Rhamnus cathartica, Berberis vulgaris, Swida sanguinea, Rosa ssp., and the non-native Ligustrum vulgare, as well as herbaceous species Laserpitium latifolium, Veronica teucrium, and Campanula persicifolia - character species of thermophilous oak forests and sparse pine woodlands of the class Quercetea pubescentis (Table 2).

The first relevé group distinguished in the study area, Filipendula vulgaris-Pinus sylvestris community, represents an overgrowing semi-natural xeric calcareous grassland that, after cessation of management, had been invaded by Pinus sylvestris. In Vegi, the dominant character species in the grassland were Filipendula vulgaris and Helictotrichon pratensis representing association Filipendulo vulgarisHelictotrichetum pratensis of the class Festuco-Brometea (Rūsiņa, 2003; 2007). Carex flacca, Pimpinella saxifraga, Brachypodium pinnatum and Knautia arvensis were constant species in this community (Table 2).

The second group of relevés represented a Quercus robur dominated forest community with ground vegetation composed of Viola mirabilis, Primula veris, Lathyrus niger and Convallaria majalis (Table 2) - species with high frequency (60\%). Also, Carex montana, a character species of thermophilous oak forests with Lathyrus niger (Roleček, 2007), was present in the ground vegetation.

Oak forest communities with the above mentioned constant species are affiliated to the thermophilous oak forest association Lathyro nigri-Quercetum roboris (Bolokhov and Solomeshch, 2003), earlier recorded in East Europe. Roleček (2007) has distinguished a similar forest community in Czechia: moderately thermophilous oak forests (as association Potentillo albae-Quercetum Libbert 1933). In Central Europe, such forests occur on deep, often heavy soils, with dominance of Quercus petraea and $Q$. robur that form open high stands. Although the syntaxonomical affiliation is rather unclear and controversial, similar thermophilous communities with $Q$. robur, the only Quercus species in Eastern Europe, occur in Ukraine and Russia (Goncharenko et al., 2020). The vegetation and site conditions recorded in Veği are like those described in Central Europe (Chytrý, 1997; Roleček, 2007), although numerous continental and subcontinental species, as well as some Mediterranean elements typical for the more southern located thermophilous oak forests, do not occur in Eastern Europe including Latvia (Goncharenko et al., 2020). These 
types of forest communities were recorded earlier also in Latvia, though they are very rare (Laiviňš, 2014).

Three character species of thermophilous oak forests (Chytrý, 1997; 2013; Chytrý and Tichý, 2008; Laivinuš, 2014), Viola mirabilis, Primula veris and Lathyrus niger, occur also in the partly overgrown xeric grassland of Filipendulo vulgaris-Pinus sylvestris, a forest pioneer stage. This points to the mutual connection of both forest communities, as they represent different subsequent succession stages, i.e., an overgrown Festuco-Brometea grassland gradually turns into a thermophilous oak community with Quercus robur and Lathyrus niger.

The presence of some species of light, warm forest fringes in the Quercus robur dominated forest, such as Origanum vulgare, Laserpitium latifolium, Veronica teucrium, Campanula persicifolia and Primula veris (Chytrý and Tichý, 2008; Laiviņš, 2014) indicates the successional relation to xeric calcareous grassland, as these species are remnants from the grassland succession stage. Currently, the frequency and abundance of these species in the oak forest is not high, as their primary habitat is warm, dry forest fringes, i.e., in Vegi they do not anymore occur in their optimum niche but can be considered as residuals of the earlier succession stage. Their presence in the oak forest suggests that in the past when the forest may have been grazed these species were perhaps present in higher abundance.

The third group of relevés described in Veǵi was composed of Corylus avellana scrub that occupied some patches in the canopy openings of an oak stand. Openings in the tree layer have appeared after falling of some older oaks. The cover of Corylus avellana exceeded $85 \%$ of the shrub cover; the ground vegetation was relatively poor in species (17 species per sample plot on average), mostly composed of shade-tolerant species of Carpino-Fagetea sylvaticae (Chytrý and Tichý, 2008; Laivin̄š, 2014), such as Mercurialis perennis, Anemone nemorosa, Paris quadrifolia, Geum urbanum, and Viburnum opulus. In such hazel-dominated openings, there is lack or negligible presence of character species of thermophilous oak forests and light, warm forest fringes. As suggested by studies in Poland and Ukraine, the colonisation of hazel that may lead to its dominance and decline of thermophilous oak forest communities, is largely related to cessation of traditional grazing in forests (JakubowskaGabara, 1996; Wolański et al., 2021).

In the boreonemoral zone, young forest stages that develop after cessation of grassland management, similarly as during forest stabilisation after catastrophic events such as clearcuts, fires, windbreaks, are dominated by the pioneer woody species, e.g., birches, aspen, pine (Tullus et al., 2013). The fast-growing, light-demanding pioneers are gradually being replaced by woody species that are more tolerant against shading and form more stable forest communities dominated by spruce and deciduous broadleaved tree species (Ruskule et al., 2016). The vegetation shift in the study area in Vegi follows the same path.
The early stage of the secondary succession during the overgrowing of Filipendulo vulgaris-Helictotrichon pratensis grassland included the establishment of Pinus sylvestris and Juniperus communis. The change at landscape level can be detected by comparing the orthophoto maps of 1994 and 2019 (Fig. 1). In the mid-1990s (Fig. 1, B), the grassland was recently abandoned; some juniper and pine clusters had already established or survived during the last years when the area was still mowed or grazed.

Later, the young forest stage transformed into Quercus robur dominated forest, although $Q$. robur may also be present at earlier stages together with pines, junipers and other shrub species. In the study site, this succession is well presented by all the above-mentioned stages in a relatively small area that were represented by a transect $300 \mathrm{~m}$ in length and $20 \mathrm{~m}$ in width across the young forest and semiopen overgrowing grassland on one end and mature oak forest on the other end. As shown by the tree age studies, the stages represent a 200 - to 250 -year period. The four youngest pine forest stages, each represented by one sample plot (Table 3), represent the last 40- to 50-year period, while the oldest succession stage, the mature oak forest, reaches an age of at least 200 years. The difference between the youngest and the oldest forest stage in the succession series is about 100-150 years. However, in Veǵi an intermediate stage between the young and mature forest was not found. Most probably, this is related to the land management until the mid-20th century, when the grasslands were intensively utilised for hay collecting and grazing (Lazdiņ̌̌, 1939; Dumpe, 1999). Thus, due to continuous grazing and mowing impact, there was no space for gradual forest development at the forest edges. Thus, it can be assumed that both the grassland and oak forest were grazed, i.e., there was regular grazing impact at the forest edge and very low chance of young tree survival. During the latest decades, cessation of grazing has, most probably, caused formation of dense hazel undergrowth in the oak forest openings that would not be possible under continuous grazing pressure (Wolański et al., 2021).

The study results suggest that in xeric grasslands the encroachment of juniper may take place earlier than the establishment of pines, but later juniper is gradually replaced by pine. In the Vegi3 sample plot, some juniper individuals were a few years older than pine trees. Early establishment of juniper may be related to the grassland management practise before cessation of grazing, as some solitaire juniper individuals in pastures may survive as "non-browsable" shrubs. However, as the management history of the study area is not known, this is an assumption not supported by historical records of the particular site. The study results show that the shrub encroachment may start both with establishment of juniper (Vegi2, Vegi3), and establishment of pine (Vegi1).

In the oldest stages of pine woodland in the study area, other woody species such as Picea abies, Populus tremula and Quercus robur were recorded. The oldest spruce Picea abies was 23 years old and was found in a mixed pine- 
juniper stand (Vegi3) established around the same time as most pines. Spruce creates extra shade and somewhat modifies the environment, making it more suitable for shadetolerant species. In the later succession stage (Vegi4), the oldest spruce was 33 years old, while in the mature oak forest (Vegi5) the maximum age of spruce reached 65 years (Table 4). In all three sample plots (Vegi1-Vegi3) where the spruce was present, the Picea abies individuals had a sparse canopy and low vitality.

The intermediate stage between the medium-aged pine forest and mature oak forest (represented by Vegi4) was characterised by the presence of young (25 years) Populus tremula individuals. Due to low light availability in the forest stand, the aspen trees are spindly, their diameter at breast height was on average $6.9 \mathrm{~cm}$, and some young aspen individuals were dead. Aspen individuals were also found in the mature oak forest, some of them dead.

The increment of trees, especially for pine, has not been linear and has varied considerably since their establishment, as it depends on different environmental drivers and the synergetic processes during woodland development.

Although confined to a small area, the study site can be considered as a representative example of the succession of xeric, thermophilous plant communities that are very rare in Latvia, as they occur in highly specific abiotic conditions. To understand the vegetation composition and its variation, as well as the succession pattern at a broader scale in Latvia, where the thermophilous oak forest communities were previously considered absent (Chytrý, 1997; Goncharenko et al., 2020), further studies at other locations are necessary.

\section{REFERENCES}

Āboliṇa, A., Piterāns, A., Bambe, B. (2016). Latvijas k̦ērpji un sūnas. Taksonu saraksts. [Lichens and Mosses. Checklist of Taxa]. Saule, Daugavpils. 245 lpp. (in Latvian).

Bolokhov, A. D., Solomets, A. I. (2003). Syntaxonomy of Forest of Russian South Nechernosemie [Болохов, А. Д., Соломец, А. И. Экологофлористическая классификация лесов южного Нечерноземья России]. Брянск. 358 pp. (in Russian).

Bulokhov, A. D., Semenischenkov, Yu. A. (2008). Communities of class Querco-Fagetea Br.-Bl. et Vlieger in Vlieger 1937 in Sudost-Desna watershied area (Bryansk region). Rastitel'nost Rosii [Булохов, А. Д., Семенищенков, Ю. А. 2008. Сообщества класса Querco-Fagetea Br.-B1. et Vlieger in Vlieger 1937 в Судость-Деснянском междуречье (Брянская область). Растительность России], No. 13, 3-13 (in Russian).

Briede, A. (2018). Atmosfēra [Atmosphere]. In: Nikodemus, O., Kḷavin̄š, M., Krišjāne, Z., Zelčs, V. (eds.) Latvija. Zeme, daba, tauta, valsts [Latvia. Land, Nature, People, Country]. LU Akadēmiskais apgāds, Rīga, 231.-267. 1pp. (in Latvian).

Chytrý, M. (1997). Thermophilous oak forests in the Czech Republic: Syntaxonomical revision of the Quercetalia pubescenti-petraeae. Folia Geobotanica \& Phytotaxonomica, 32 (3), 221-258.

Chytrý, M. (Ed.) (2013). Vegetace České republiky. 4. Lesni a křovinná vegetace [Vegetation of the Czech Republic 4. Forest and Scrub Vegetation]. Academia, Praha, 551 pp. (in Czech).

Chytrý, M., Tichý, L. (2008). Diagnostic, constant and dominant species of vegetation classes and alliances of the Czech Republic: a statistical revi- sion. Folia Facultatis Scientiarum Naturalium Universitatis Masarykianae Brunensis. Biologia, 108, 1-23.

Dierschke, H. (1994). Pflanzensoziologie. Verlag Eugen Ulmer, Stuttgart. 683 pp. (in German).

Dumpe, L. (1999). Meža izmantošanas attīstība Latvijā [Development of forest use in Latvia]. In: Strods, H. (Ed.). Latvijas mežu vēsture lìdz 1940. gadam [History of Forest in Latvia Until 1940]. WWF (Pasaules Dabas fonds), Rīga, 305.-357. lpp. (in Latvian).

Eberhards, G. (2013). Upju ieleju veidošanās un mūsdienu gultnes procesi dienvidaustrumu Baltijā [Formation of River Valleys and Modern Processes in Valley Formation in the Southeastern Baltics]. LU Akadēmiskais apgāds, Rīga. 212 lpp. (in Latvian)

Ellenberg, H., Weber, H. E., Düll, R., Wirth, V., Werner, W., Paulissen, D. (1992). Zeigerwerte von Pflanzen in Mitteleuropas. Scripta Geobotanica, 18, 1-258 (in German).

Gavrilova, G̦., Šulcs, V. (1999). Latvijas vaskulāro augu flora. Taksonu saraksts [Vacular Plant Flora in Latvia. Checklist]. Rīga. 134 pp. (in Latvian).

Goncharenko, I., Semenishchenkov, Y., Tsakalos, J. L., Mucina, L. (2020). Thermophilous oak forests of the steppe and forest-steppe zones of Ukraine and Western Russia. Biologia, 75, 337-353.

Jakubowska-Gabara, J. (1996). Decline of Potentillo albae-Quercetum Libb. 1933 phytocoenoses in Poland. Vegetatio, 124, 45-59.

Jermacāne, S., Laiviṇš, M. (2001). Latvijā aprakstīto augu sabiedrību sintaksonu saraksts [The checklist of vegetation syntaxa in Latvia]. Latvijas Veǵetācija, 4, 115-132

Krievāns, M., Soms, J., Strautnieks, I., Zelčs, V. (2018). Upju ieleju sistēmas un nozīmīgākās upju ielejas [River valley systems and the most significant river valleys]. In: Nikodemus, O., Kḷaviņš, M., Krišjāne, Z., Zelčs V. (eds.) Latvija. Zeme, daba, tauta, valsts [Latvia. Land, Nature, People, Country]. LU Akadēmiskais apgāds, Rīga, 115.-130. lpp. (in Latvian).

Laiviņš, M. (2014). Latvijas meža un krūmāju augu sabiedrības un biotopi [Communities and habitat types of forests and scrubs in Latvia]. Mežzinātne, 28 (61), 6-38 (in Latvian).

Lazdiņš, L. (1939). Lopu ganīšana mežā [Pasturing in forest]. Sēta un Druva, 25, 414-415.

Liepa, I. (1974). Biometrija [Biometry]. Zvaigzne, Rīga. 336 lpp.

McCune, B., Grace, J. B. (2002). Analysis of Ecological Communities. MjM Software Design, Glenden Beach, Oregon. 300 pp.

McCune, B., Mefford, M. J. (2011). PC-ORD. Multivariate analysis of Ecological Data, Version 6.0 for Windows. MjM Software, Gleneden Beach, Oregon.

Mucina, L., Grabherr, G., Walnöfer, S. (Hrgb.) (1993). Die Pflanzengesellschaften Östererreichs. Teil III. Wälder und Gebüsche. Gustav Fischer Verlag, Jena, Stuttgart, New York. 353 pp.

Mucina, L., Bültmann, H., Dierssen, K., Theurillat, J.-P., Raus, T., Čarni, A., Śumberová, K., Willner, W., Dengler, J., García, R. G. et al. (2016). Vegetation of Europe: Hierarchical floristic classification system of vascular plant, bryophyte, lichen, and algal communities. Appl. Veg. Sci., 19, 3-264.

Peel, M. C., Finlayson, B. L., McMahon, T. A. (2007). Updated world map of the Köppen-Geiger climate classification. Hydrol. Earth Syst. Sci., 11, 1633-1644.

Priede, A. (2009). Dynamics of non-native flora: Changes over the last decades in the Abava River valley. Latvijas Universitâtes Raksti. Zemes un Vides zinātnes, 724, 89-108.

Roleček, J. (2007). Formalized classification of thermophilous oak forests in the Czech Republic: What brings the Cocktail method? Preslia, 79 (1), $1-21$ 
Ruskule, A., Nikodemus, O., Kasparinskis, R., Prižavoite, D., Bojāre, D., Brūmelis, G. (2016). Soil-vegetation interactions in abandoned farmland within the temperate region of Europe. New Forests, 47, 587-605.

Rūsina, S. (2003). Dry calcareous grassland communities (Filipendula vulgaris-Helictotrichon pratense) in western and central Latvia. Ann. di Bot., III, 91-104.

Rūsiņa, S. (2007). Latvijas mezofīto un kserofīto zālāju daudzveidība un kontaktsabiedrības [Diversity and Contact Communities of Mesophytic and Xerophytic Grasslands in Latvia]. Latvijas Veǵetācija, 12, 1-366.

Stinkule, A., Stinkulis, G. (2013). Latvijas derīgie izrakteni [Resources of Subterranean Depths in Latvia]. LU Akadēmiskais apgāds, Rīga, 187 lpp.

Strautnieks, I. (2020). Abavas senleja [Abavas ancient valley]. Nacionālā enciklopédija, https://enciklopedija.lv/skirklis/59207-Abavas-senleja (accessed 13.01.2021).

Szygendowski, A., Brzeg, A. (2015). Thermophilous fringe communities as an indicator of vegetation changes: A case study of the "Murawy Dobromierskie" steppe reserve (Poland). Biodivers. Res. Conserv., 40, 77-104.

Received 8 March 2021

Accepted in the final form 3 August 2021
Tabaka, L. V., Klavina, G. B. (1981). Abava River Valley. Flora of Protected Areas in Latvia [Табака, Л. В., Клявиня, Г. Б. Долина реки Абава. Флора охраняемых территорй Латвии]. Zinātne, Rīga. 131 pp. (in Russian)

Temnikova, N. S. (1958). Climate of the Latvian SSR [Темникова, H. C. Климат Латвийской ССР]. Izdatelstvo AN Latviyskoy SSR, Riga, 232 pp.

Tullus, T., Tullus, A., Roosaluste, E., Kaasik, A., Lutter, R., Tullus, H (2013). Understorey vegetation in young naturally regenerated and planted birch (Betula spp.) stands on abandoned agricultural land. New Forests, $\mathbf{4 7}$ 587-605.

Willner, W., Grabherr, G. (2007). Die Wälder und Gebüsche Osterreichs. Ein Bestimmungswerk mit Tabellen. Textband. Elsevier GmbH, München. $302 \mathrm{~S}$.

Wolański, P., Bobiec, A., Ortyl, B., Makuch-Pietraś, I., Czarnota, P., Ziobro, J., Korol, M., Havryliuk, S., Paderewsk, J., Kirby, K. (2021). The importance of livestock grazing at woodland-grassland interface in the conservation of rich oakwood plant communities in temperate Europe. Biodivers. Conserv., 30, 741-760.

\section{SAUSU KAḶKAINU ZĀLĀJU TRANSFORMĀCIJA TERMOFĪLOS OZOLU MEŽOS: ABAVAS SENIELEJAS PIEMĒRS}

Sausu kaḷkainu zālāju augu sabiedrību aizaugšana, dabiski pārveidojoties platlapju mežu sabiedrībās, Latvijā ir maz pētīta. Maz zināms par sausu kalłķainu Festuco-Brometea klasei piederošu zālāju sukcesijas gaitu, tiem aizaugot un pārveidojoties kvaziklimaksa termofīlo ozolu Quercetea pubescentis klasei piederošu ozolu mežu sabiedrībās. Šādas ozolu mežu sabiedrības Latvijā ir l̦oti reti sastopamas. Rakstā atspoguḷoti ǵeobotāniska pētījuma rezultāti, kas ietvēra augsnes, koku vecuma un telpiskā izvietojuma, kā arī veǵetācijas sugu sastāva un vides faktoru analīzi dažādās sukcesijas stadijās. Pētījums tika īstenots Abavas senielejā Rietumlatvijā — teritorijā, ko raksturo sugām bagāti sausi kalkaini Festuco-Brometea klases zālāji, kā arī sastopamas termofīlo ozolu mežu sabiedrības ar parasto ozolu Quercus robur, bet starpstadijās sastopamas jaunas līdz vidēja vecuma kadiḳu Juniperus communis un parastās priedes Pinus sylvestris audzes. 